\title{
Aeronave remotamente pilotada de baixo custo no estudo de plantas invasoras em áreas de cerrado
}

\author{
Dhonatan Diego PESSI ${ }^{*}$, Jefferson Vieira JOSÉ 2 , Camila Leonardo MIOTO${ }^{1}$, \\ Normandes Matos da SILVA ${ }^{1}$
}

\begin{abstract}
${ }^{1}$ Programa de Pós-Graduação em Gestão e Tecnologia Ambiental, Universidade Federal de Rondonópolis, Rondonópolis, MT, Brasil. (ORCID: *; 0000-0002-6951-9527; 0000-0001-8934-6507)

${ }^{2}$ Centro Multidisciplinar, Campus Floresta, Universidade Federal do Acre, Cruzeiro do Sul, AC, Brasil. (ORCID: 0000-0003-1384-0888) *E-mail: dhonatan.pessi@gmail.com (ORCID: 0000-0003-0781-785X)
\end{abstract}

Recebido em: 24/05/2019; Aceito em: 22/07/2019; Publicado em: 04/02/2020.

\begin{abstract}
RESUMO: O objetivo dessa pesquisa é analisar se o método $\mathrm{CH}_{\mathrm{IS}}$ (Canopy Height Invasive Species) representa uma rotina de classificação assertiva na identificação de espécies invasoras a partir de imagens RGB em área de Cerrado com evidência de perturbação. A metodologia empregada foi a produção dos modelos de elevação MDS (Modelo Digital de Superfície) e MDT (Modelo Digital do Terreno) a partir das imagens coletadas em campo com drone e posteriormente processadas no software PhotoScan. A produção do $\mathrm{CH}_{\mathrm{IS}}$ foi a partir da subtração dos modelos MDS e MDT. Para aferir a precisão do modelo $\mathrm{CH}_{\mathrm{IS}}$ foram gerados dois modelos convencionais para comparação: classificação não supervisionada K-means e índice de vegetação NGRDI (Normalized Red-Green Difference Index). A comparação entre os modelos se deu em duas áreas amostrais escolhidas de forma não aleatória. Ao final foi aplicado teste de acurácia, correlação e Cohen’s Kappa. Os resultados demonstram que o modelo $\mathrm{CH}_{\mathrm{IS}}$ obteve os melhores resultados na identificação de espécies invasoras quando comparado com os modelos K-means e NGRDI. Os testes de acurácia para o modelo $\mathrm{CH}_{\mathrm{IS}}$ na área amostral 1 e 2 foi de 0,973 e 0,9 respectivamente; K-means 0,209 e 0,6; NGRDI 0,795 e 0,518. O modelo $\mathrm{CH}_{\mathrm{IS}}$ demonstrou ser promissor na identificação de espécies invasoras em áreas perturbadas quando comparado com modelos convencionalmente usados.
\end{abstract}

Palavras-chave: ARP; gestão ambiental; sensoriamento remoto; $\mathrm{CH}_{\mathrm{IS}}$.

Remotely piloted aircraft (drone) of low cost in the invasive species study in Cerrado areas

\begin{abstract}
The objective of this research is to analyze if the $\mathrm{CH}_{\mathrm{IS}}$ (Canopy Height Invasive Species) method represents an assertive classification routine in the identification of invasive species from RGB images in Cerrado area with evidence of disturbance. The methodology used was the production of the DSM (Digital Surface Model) and DTM (Digital Terrain Model) elevation models from the images collected in the drone field and later processed in the PhotoScan software. The production of the $\mathrm{CH}_{\mathrm{IS}}$ was based on the subtraction of the DSM and DTM models. To verify the accuracy of the $\mathrm{CH}_{\mathrm{IS}}$ model two conventional models were generated for comparison: unsupervised K-means classification and NGRDI (Normalized Red-Green Difference Index) vegetation index. The comparison between the models occurred in two sample areas chosen in a non-random manner. At the end, it was applied test of accuracy, correlation and Cohen's Kappa. The results demonstrate that the $\mathrm{CH}_{\text {IS }}$ model obtained the best results in the identification of invasive species when compared with the K-means and NGRDI models. The accuracy tests for the $\mathrm{CH}_{\mathrm{IS}}$ model in sample area 1 and 2 were 0,973 and 0,9 respectively; K-means 0,209 and 0,6; NGRDI 0,795 and 0,518. The $\mathrm{CH}_{\text {Is }}$ model has been shown to be promising in the identification of invasive species in disturbed areas when compared to conventionally used models.
\end{abstract}

Keywords: RPA; environmental management; remote sensing; $\mathrm{CH}_{\mathrm{IS}}$.

\section{INTRODUÇÃO}

As aeronaves remotamente pilotadas (ARP), popularmente conhecidas como drones, tiveram uma evolução rápida na última década, tanto para fins militares como para uso civil (IVOSEVIC et al., 2015). O processamento de dados derivados de ARP está em rápida evolução e é utilizado em uma ampla gama de pesquisas geoespaciais, incluindo a gestão de recursos naturais (GINI et al., 2018). As ARPs de baixo custo apresentam boa relação custo versus benefício, para a pesquisa e monitoramento ambiental devido à sua facilidade de uso, versatilidade e sua capacidade de coletar dados de áreas, que de outra forma são de difícil acesso (EVANS et al., 2015; GINI et al., 2018; STARK et al., 2018). Em função da baixa altitude operacional de voo realizada em missões com ARP, as imagens aéreas capturadas são de altíssima resolução espacial (PENG et al., 2015; STARK et al., 2018). Além disso, o baixo custo dessas plataformas, juntamente com a variedade de sensores disponíveis, torna a ARP adequada para ser utilizada em muitas situações em que um meio tradicional (satélites e 
aviões tripulados) seria de alto custo financeiro (STARK et al., 2018).

Os sensores mais comuns acoplados às ARPs são câmeras digitais convencionais que registram dados na faixa do espectro visível (RGB), oferecendo vantagens sobre outras plataformas de sensoriamento remoto. Estes sensores possuem capacidade de gerar imagens de alta resolução em escalas temporais flexíveis, tornando as ARPs adequadas para preencher a lacuna entre os dados contidos em amostras de campo de pequena escala e a necessidade de compreensão de padrões da paisagem em áreas extensas (ZHANG et al., 2015; STARK et al., 2018).

Em particular, os sistemas de aeronaves remotamente pilotadas (SARP) já deram provas de serem plataformas apropriadas para o planejamento urbano, agrícola e ambiental, dentre outras finalidades (KANEKO; NOHARA, 2014). Configurações compostas por câmeras digitais padrão e multiespectrais propiciaram resultados promissores em estudos de florestas do Mediterrâneo (PENG et al., 2015), áreas de pastagens áridas (RANGO et al., 2009; LALIBERTE et al., 2011), leitos de musgo antártico (LUCIEER et al., 2012; TURNER et al., 2014) e plantas daninhas aquáticas (GOKTOGAN et al., 2010). As ARPs têm potencial para mapear e monitorar ecossistemas ameaçados e de espécies invasoras ou dominantes (HARDIN et al., 2007; BAENA et al., 2017), e também podem ser usadas na coleta de informações durante desastres ambientais, no gerenciamento e monitoramento de rios e aterros, e na medição e levantamento topográfico (KANEKO; NOHARA, 2014). Além disso, existe o potencial deste sistema para aplicações ambientais e de conservação, que incluem mapeamento quase em tempo real da cobertura da terra local e monitoramento de atividades florestais ilegais, como por exemplo, extração de madeira e incêndios (KOH; WICH, 2012).

Devido ao baixo custo e ao aumento da capacidade das ARPs, em termos de produção de ortomosaicos e de modelos digitais da superfície, pesquisadores começaram a explorar essa tecnologia para mapeamento de espécies

invasoras (MICHEZ et al., 2016; DVORÁK et al., 2015; HUNG et al., 2014; WAN et al., 2014; KNOTH et al., 2013; PEÑA et al., 2013; ZAMAN et al., 2011). Trabalhos anteriores sobre o mapeamento de espécies invasoras com ARP exploraram produtos baseados na classificação de pixels (WAN et al., 2014; ZAMAN et al., 2011), detecção de objetos (MICHEZ et al., 2016; HUNG et al., 2014; KNOTH et al., 2013; PEÑA et al., 2013) e métodos de classificação por meio da altura do dossel (MATESE et al., 2017; STROPPIANA et al., 2018; ZILIANI et al., 2018; VILJANEN et al., 2018; SÁ et al., 2018; MARTIN et al., 2018) para identificar as espécies de interesse das imagens adquiridas.

Nesse sentido, o objetivo deste estudo consiste em analisar se o método $\mathrm{CH}_{\mathrm{IS}}$ (Canopy Height Invasive Species) representa uma rotina de classificação assertiva na identificação de espécies invasoras a partir de imagens RGB em área de Cerrado com evidência de perturbação.

\section{MATERIAL E MÉTODOS}

\section{1. Área de estudo}

A área de estudo localiza-se na Universidade Federal de Rondonópolis (Figura 1), em Rondonópolis, Mato Grosso, Brasil. O local estudado apresenta elementos de vegetação remanescente de Cerrado stricto sensu, locais com solo exposto e áreas com avançada presença de espécies invasoras, predominantemente a espécie Urochloa ssp. A área estudada abrangeu três hectares. A característica climática local é definida como Cwa (clima subtropical úmido) com média anual em precipitação de $1500 \mathrm{~mm}$ e na temperatura de $25^{\circ} \mathrm{C}$ (SOUZA et al., 2013; PEEL et al., 2007).
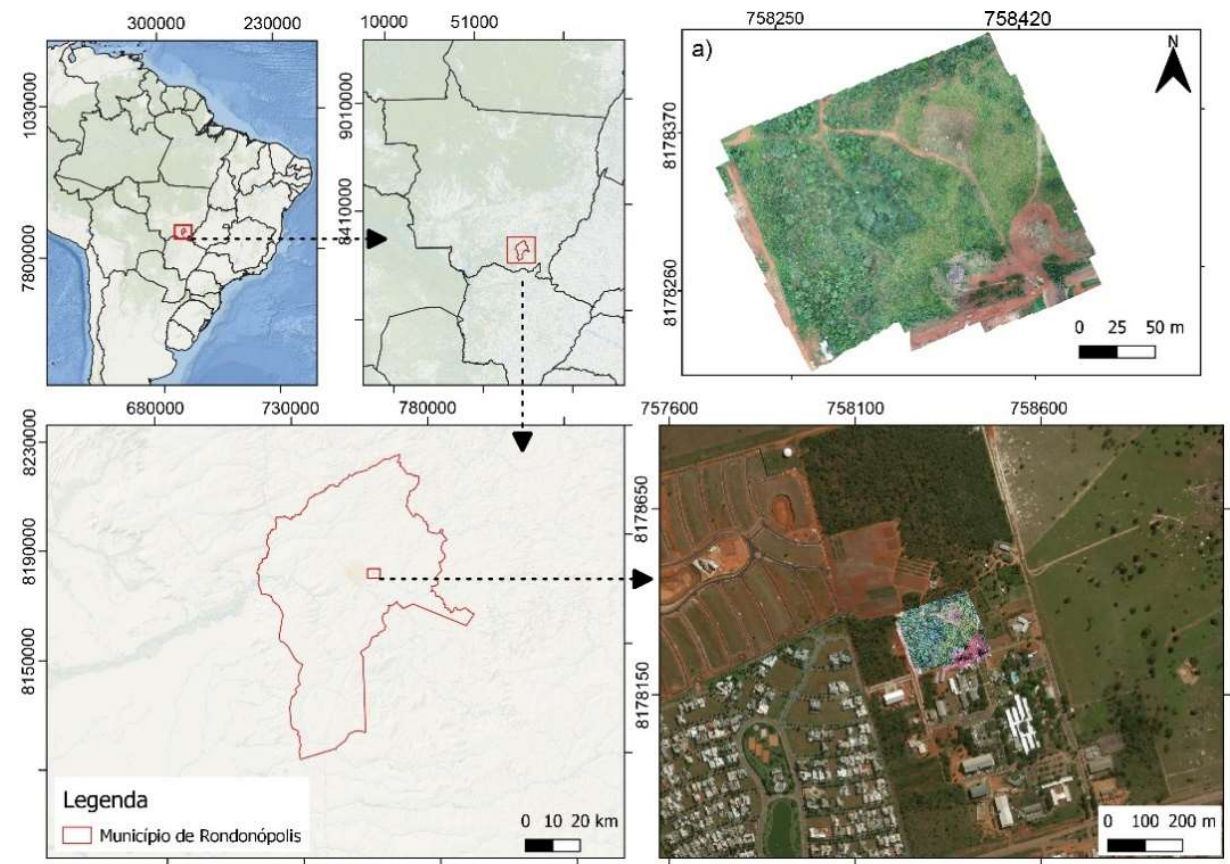

Figura 1. Localização geral da área de estudo. Em a) sítio experimental com o ortomosaico de imagens obtidas pelo ARP. Sistema de Coordenadas UTM, SIRGAS 2000, 21S.

Figure 1. General location of the study area. In a) experimental site with the ortomosaico of images obtained by ARP. Coordinate System UTM, SIRGAS 2000, 21S. 


\subsection{ARP e sensor}

A plataforma ARP usada neste estudo foi um multirotor DJI Phantom 4 Pro. A ARP Phantom 4 Pro pode ser conectada via datalink a um controle remoto e dispositivo móvel (telefone celular ou tablete), tornando possível monitorar o status do voo da ARP e enviar instruções da missão do trajeto de voo via aplicativo. Nesta pesquisa utilizou-se da plataforma DroneDeploy (disponível em: https://support.dronedeploy.com/lang-pt-BR) para planejamento dos parâmetros de voo e coleta de imagens. Os detalhes das especificações da plataforma ARP usada neste estudo estão disponíveis no manual do usuário (https://dl.djicdn.com/downloads/phantom_4_pro/20170 719/Phantom_4_Pro_Pro_Plus_User_Manual_ES.pdf).

\subsection{Aquisição e processamento das imagens RGB}

A ARP foi configurada para voar automaticamente sobre a área de estudo, a partir da execução de missão automatizada, enquanto rastreava waypoints de acordo com o trajeto de voo pré-programado gerado no programa DroneDeploy. Uma sequência de imagens sobrepostas foi coletada em cada missão de voo para cobrir toda a área estudada.

A partir das imagens RGB adquiridas em campo pela ARP, foi gerado o ortomosaico por meio do programa Agisoft Photoscan Profissional (version 1.4.0, Agisoft, St. Petersburg, Russia). Uma malha de pontos foi computada a partir da nuvem densa de pontos 3D e os valores de pixel de cada imagem foram então projetados na malha de pontos para criar um ortomosaico.

\subsection{Conferência de dados em campo}

Coletou-se dados de referência compostos por pontos e medidas, rotulados como Urochloa ssp. A coleta dos pontos foi realizada a partir de um GPS Garmin 76CSx. Ao todo foram coletados 37 pontos de referência em campo (Figura 2). Já a coleta das medidas da altura das espécies invasoras foi por meio de trena métrica para estabelecer a altura das espécies invasoras em cada ponto de coleta para diferenciá-las da vegetação remanescente na validação dos dados da altura do dossel das espécies invasoras $\left(\mathrm{CH}_{\mathrm{IS}}\right)$.

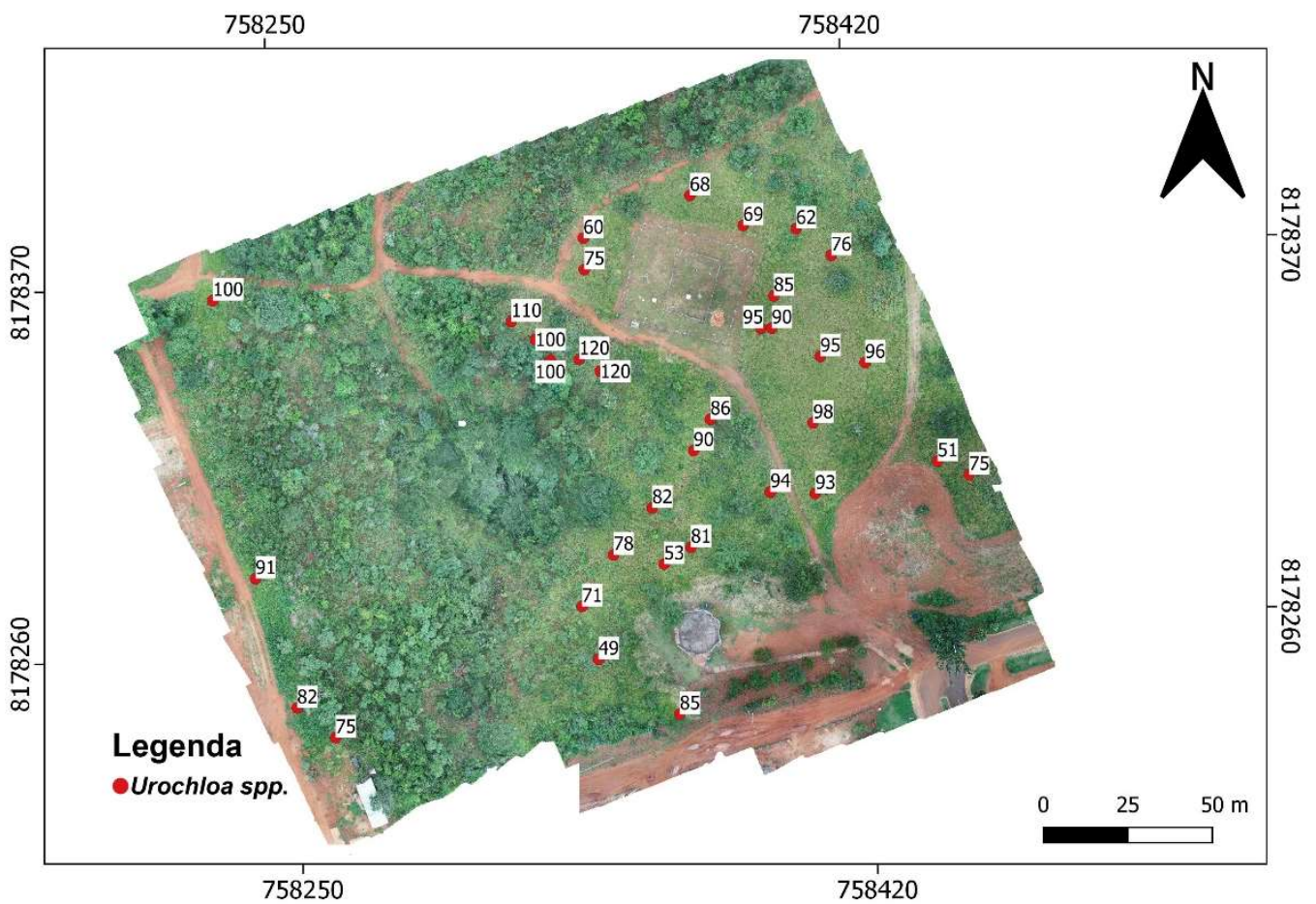

Figura 2. Conjunto de dados de referência extraído em campo. Pontos em vermelho se referem aos locais com presença de Urochloa ssp. Os valores informados para cada ponto se referem a altura em centímetros $(\mathrm{cm})$.

Figure 2. Set of reference data extracted in the field. Points in red refer to locations with Urochloa ssp. The values reported for each point refer to the height in centimeters $(\mathrm{cm})$.

\section{5. Índices de vegetação (IV)}

Dadas as configurações de banda específicas da câmera, o IV utilizado deve ser aquele que atenda as configurações de banda RGB. O índice escolhido para este estudo foi o NGRDI, pois fornece melhores resultado visualmente quanto à classificação por meio de imagens RGB (GITELSON et al., 2002). O índice NGRDI fornece indicações da variabilidade espectral dos dosséis da vegetação no plano horizontal (LI et al., 2016). Este índice de vegetação foi originalmente proposto devido ao seu potencial na discriminação espectral de espécies invasoras em cultivos
(BARRERO; PERDOMO, 2018; DAVID; BALLADO, 2016). O cálculo do índice NGRDI assim como a plotagem foi realizado no software R 3.5.1 (R CORE TEAM, 2018) com os pacotes raster (HIJMANS, 2019), grDevices (R CORE TEAM, 2018) e RColorBrewer (NEUWIRTH, 2014).

\subsection{Classificação não supervisionada}

O algoritmo usado neste estudo para classificação não supervisionada foi o k-means, por ser um algoritmo comumente usados com bons resultados na classificação não supervisionada (VISHWANATH et al., 2016). 
Inicialmente, o algoritmo avalia cada dado em cluster aleatoriamente e localiza o centroide de cada cluster. Em seguida, o algoritmo atribui pontos de dados para o cluster cujo centroide está mais próximo; e calcula novo centroide de cada cluster. Estas duas etapas continuam a ser executadas até que a variação intra-classe não possa ser reduzida ainda mais. A variação intra-cluster é calculada como a soma da distância euclidiana entre os pontos de dados e seus respectivos centroides de cluster (VISHWANATH et al., 2016). A classificação K-means foi realizado no software $R$ 3.5.1. Os pacotes utilizados foram raster e cluster (MAECHLER et al., 2018).

\subsection{Altura da copa de espécies invasoras $\mathbf{C H}_{I S}$}

A altura do dossel de espécies invasoras $\left(\mathrm{CH}_{\mathrm{IS}}\right.$ Canopy Height Invasive Species) foi estimada pela diferença matemática do Modelo Digital de Superfície (MDS) e do Modelo Digital do Terreno (MDT). O MDS foi gerado no software Agisoft Photoscan Profissional versão 1.4.0, a partir dos valores das medidas do GNSS (Global Navigation Satellite System) localizadas no EXIF das imagens capturadas pelo Phantom 4 Pro em campo.

O MDS é obtido a partir da nuvem densa de pontos. A nuvem densa de pontos é uma função a qual a partir dos dados de coordenadas geográficas inseridas nas fotos, produz um modelo 3D. A produção do MDT consistiu em classificar a nuvem densa de pontos no Agisoft Photoscan, para excluir os elementos acima do solo. Feito este procedimento, o passo seguinte consistiu em aplicar um "smoth mesh", função do PhotoScan para corrigir erros e deixar a malha mais refinada. Com a malha pronta, foi realizado o procedimento de "build DEM" nas ferramentas do PhotoScan. Após esse procedimento, foi utilizado a malha (points of ground) como base para o cálculo do MDS para gerar o MDT.

Ao final do processo de produção dos modelos MDS e MDT, foi gerado um relatório no PhotoScan de erros de acurácia posicional nos eixos $\mathrm{X}, \mathrm{Y}$ e $\mathrm{Z}$ para saber se houve muita diferença nos valores dos eixos comparado com os dados avaliados em campo, uma vez que esta pesquisa simulou uma rotina sem a correção de erros com RTK (GPS geodésico). Sendo assim, a pesquisa forneceu dados de calibração do modelo apenas com o sistema GNSS da ARP.

Para calcular o modelo de altura de dossel (CHM Canopy) Height Model) foi necessário calcular a subtração do MDT e do MDS no software R. A partir do CHM foi possível identificar os diferentes estratos da vegetação e selecionar apenas as informações que estivessem no limite da altura estabelecida para as espécies invasoras (modelo $\mathrm{CH}_{\mathrm{IS}}$ ), que neste caso foram definidos como valores mínimos de $49 \mathrm{~cm}$ e valores máximos de $110 \mathrm{~cm}$ para Urochloa ssp., conforme dados levantados em campo. Entretanto, também optou em definir valores diferentes aos observados em campo, para mais e para menos, para considerar as possíveis diferenças no modelo (erros), desta maneira, foi informado ao programa para selecionar valores que compreendam desde $20 \mathrm{~cm}$ até $140 \mathrm{~cm}$.

A comparação do modelo $\mathrm{CH}_{\mathrm{IS}}$ entre os modelos $\mathrm{k}$ means e NGRDI foi através de duas áreas amostrais, uma vez que não era necessário realizar a comparação em toda a área, facilitando o trabalho comparativo e o processamento de dados. Foram selecionadas duas áreas específicas que possuíam todos os elementos necessários para comparação e que não fugiam da finalidade deste estudo.
2.8. Avaliação da precisão: teste de acurácia, correlação e Cohen's Kappa

Para calibrar e validar a classificação do modelo $\mathrm{CH}_{\mathrm{IS}} \mathrm{e}$ dos modelos comparativos k-means e NGRDI, foi necessário gerar um raster de classificação supervisionada a partir do ortomosaico da área estudada. Essa rotina resultou em duas classes: ausência de espécies invasoras (aqui pode ser considerado a vegetação remanescente) sendo valor 1 e a presença de espécies invasoras como valor 2 . Os demais modelos $\left(\mathrm{CH}_{\mathrm{IS}}\right.$, k-means e NGRDI) foram todos reclassificados em apenas duas classes (presença ou ausência de espécies invasoras) assim como os dados de referência. A reclassificação foi realizada a partir da interpretação dos resultados dos modelos, identificando quais valores se referiam às espécies invasoras e quais valores eram atribuídos a outras classes.

O teste visou comparar apenas duas áreas amostrais (Figura 3). A técnica consistiu em recolher 1000 pontos amostrais, os quais foram gerados aleatoriamente (os pontos são os mesmos para todos os modelos avaliados) para cada área amostral da área de estudo. $\mathrm{O}$ recolhimento dos pontos amostrais foi realizado no software $\mathrm{R}$ 3.5.1. Esta é uma prática comum em sensoriamento remoto quando imagens de alta resolução estão disponíveis e a distinção entre as classes é clara (FENG et al., 2015; LEHMANN et al., 2017; MÜLLEROVÁ et al., 2017; SÁ et al., 2018) como é o caso deste estudo. A precisão da estimativa foi quantificada usando Coeficientes de Correlação de Spearman (SCC), Precisão Geral (OAR) e Coeficiente Kappa de Cohen (K).

A identificação visual dos pontos de calibração/validação foi realizada usando o software R. 3.5.1. Pacotes usados para: (i) reclassificação dos dados raster, classificação supervisionada, extração dos dados para análise de acurácia foram usados os pacotes raster, rgdal (BIVAND, 2018) e shapefiles (STABLER, 2013); (ii) testes de acurácia e índice Kappa foram usados os pacotes rcompanion (MANGIAFICO, 2019), caret (KUHN, 2018) e forecast (HYNDMAN et al., 2019)

\section{RESULTADOS}

\subsection{Modelo de Altura do Dossel (CHM)}

Os valores das alturas compreendem a partir do nível do mar, que foram 281 a 298 metros no MDS e 281 a 288 metros no MDT (Figura 4).

O MDT, em uma primeira análise, destaca-se por apresentar uma diferença anormal nas medidas de altitude, localizada na parte superior direita, uma diferença entre 3 a 5 metros. Estes valores podem estar relacionados aos erros no algoritmo que interpola os pontos homólogos e as imagens para produção do MDT. Além disso, pode também estar relacionada com a menor sobreposição de fotografias (menos pontos homólogos) principalmente nas bordas da área de estudo, onde ocorre uma menor sobreposição (Figura 5).

As altitudes da vegetação variaram desde valores negativos $(-4 \mathrm{~m})$ até valores positivos $(14 \mathrm{~m})$ (Figura 6), contudo, considerou-se vegetação apenas os valores positivos que compreenderam desde 0 metros até 14 metros. $\mathrm{Na}$ Figura 7 é representado o modelo em 3D da altura do dossel. 


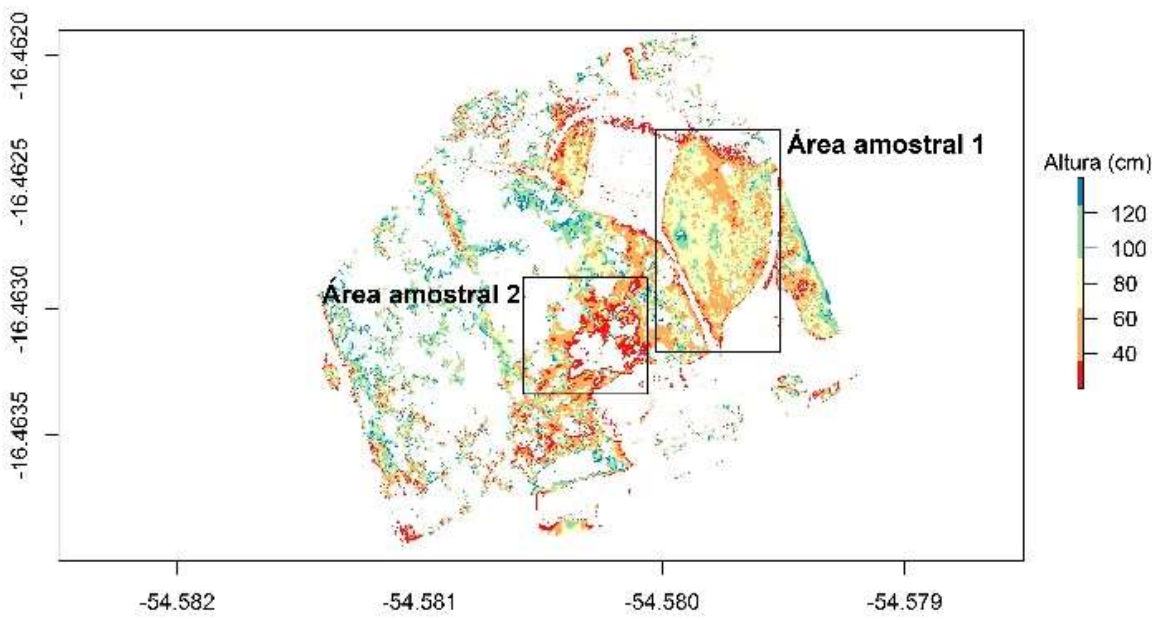

Figura 3. Localização das áreas amostrais usadas no teste de precisão dos modelos comparados neste estudo. Figure 3. Location of the sample areas used in the precision test of the models compared in this study.
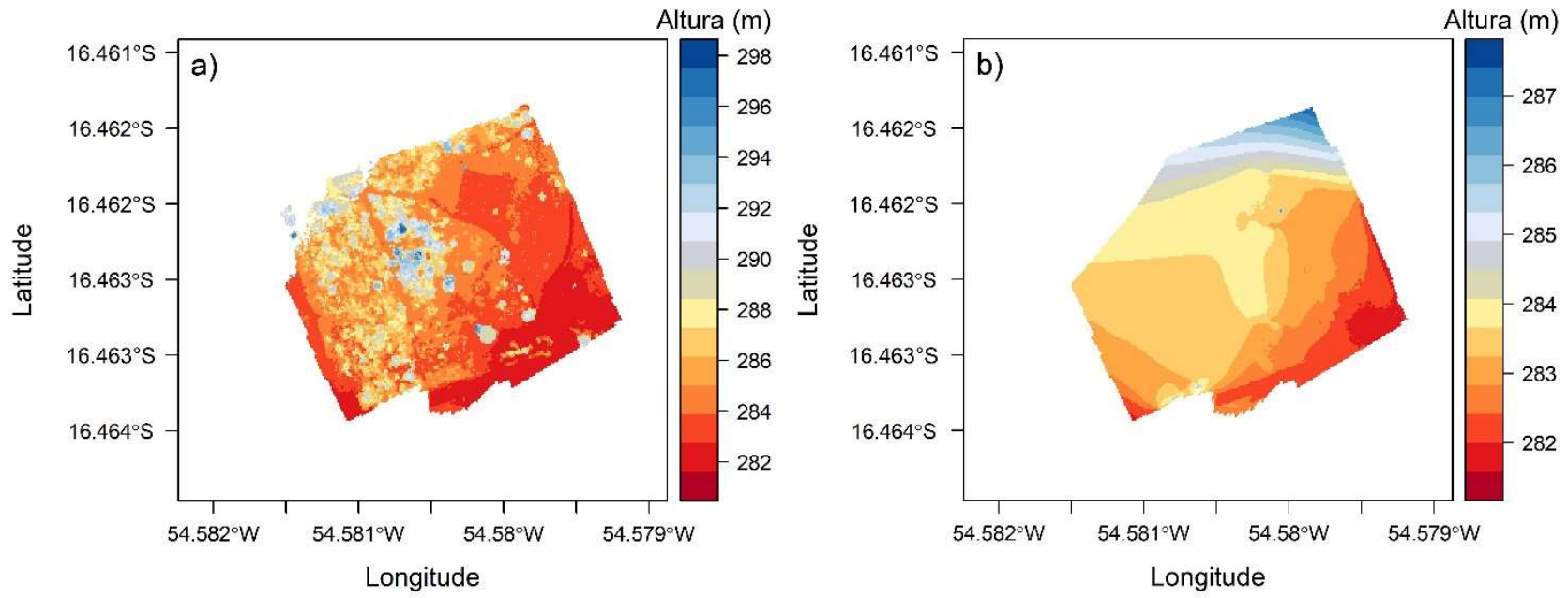

Figura 4. Modelo digital de superfície (MDS) (a); modelo digital do terreno (MDT) gerados a partir de mosaico de imagens aéreas derivadas de aeronave remotamente pilotada (b). Os valores de altimetria são considerados a partir do nível do mar

Figure 4. Digital surface model (DSM) (a); digital terrain model (DTM) generated from mosaic of aerial images derived from remotely piloted aircraft (b). Altimetry values are considered from sea level.

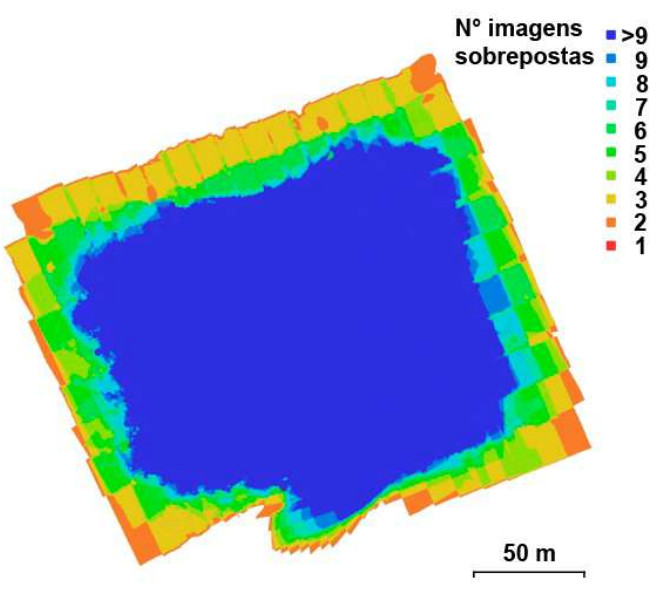

Figura 5. Locais da câmera e sobreposição das imagens. Figure 5. Camera locations and image overlay.

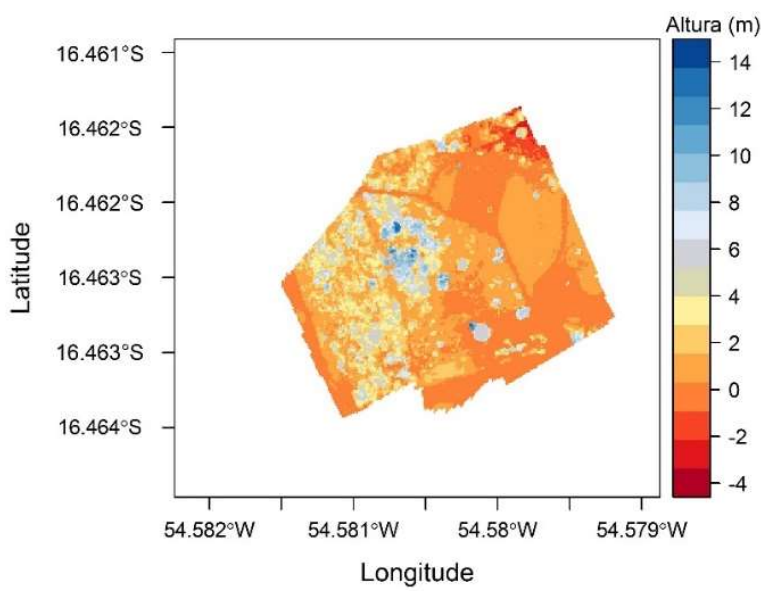

Figura 6. Altura do dossel da vegetação (CHM) presente na área de estudo.

Figure 6. Height of the vegetation canopy (CHM) present in the study area. 


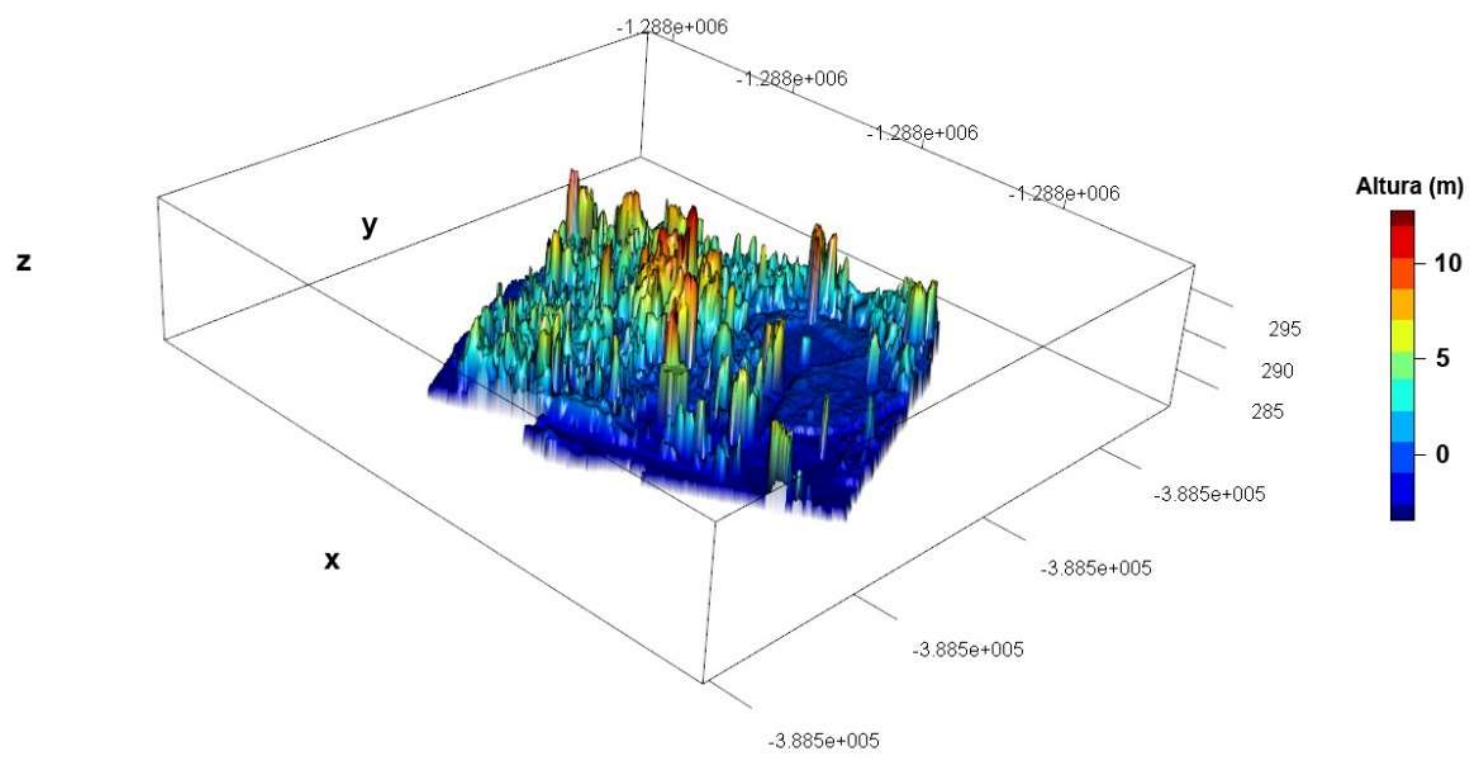

Figura 7. Modelo 3D da altura do dossel da vegetação presente na área de estudo.

Figure 7. 3D model of canopy height of the vegetation present in the study area.

$\mathrm{Na}$ Figura 8, apresenta-se o CHM com as medidas definidas e representadas com diferentes cores. A escala de cores representa os valores referentes a escala de $0,00 \mathrm{~m}$ até $15 \mathrm{~m}$. Estes valores se referem as diferentes alturas de dossel da vegetação presentes na área analisada. A escala a partir do valor de $0,00 \mathrm{~m}$, compreendendo a cor branca, refere-se a classe de solo exposto. Este valor pode variar até os $15 \mathrm{~cm}$, podendo ser considerado solo exposto ou vegetação de pequenas proporções. A partir destes resultados, numa primeira análise, não é possível separar o solo exposto de espécies invasoras de pequena altura $(0,20 \mathrm{~cm})$. Para tanto é necessário realizar uma seleção dos valores de interesse a partir do modelo CHM que corresponda as espécies invasoras $\left(\mathrm{CH}_{\mathrm{IS}}\right)$.

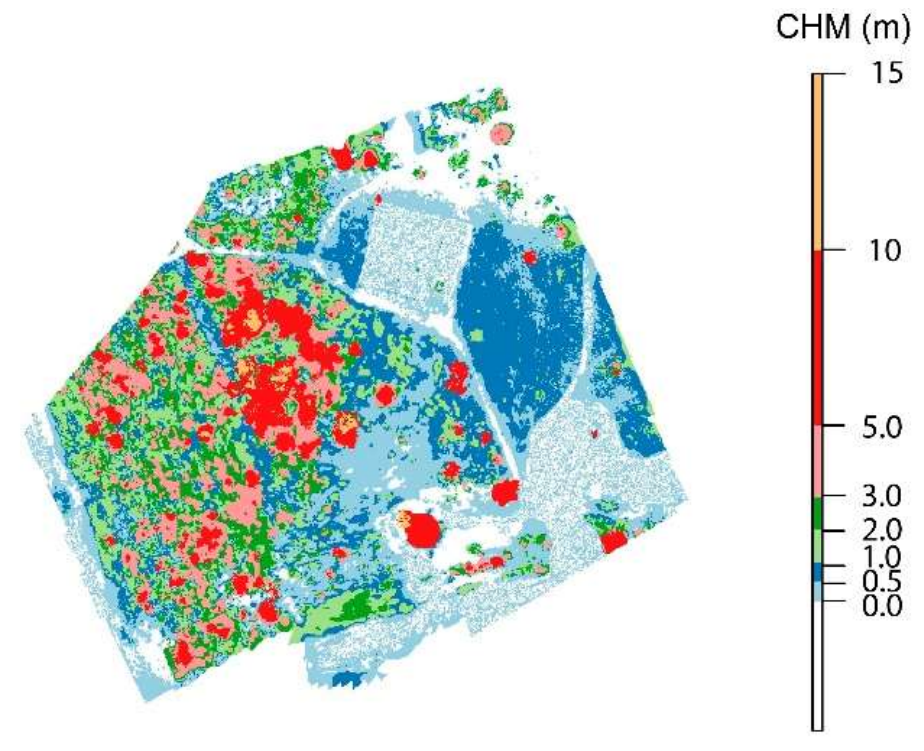

$\mathbf{A}$

$100 \mathrm{~m}$

Figura 8. CHM com escala de valores referentes à altura do dossel. Figure 8. CHM with scale of values referring to the canopy height.

\subsection{Classificador $k$-means $\mathrm{e}$ índice de vegetação NGRDI}

O modelo de classificação não supervisionada K-means gerou três diferentes classes, que foram numeradas em ordem crescente, e o índice de vegetação NGRDI gerou os valores na escala de 1 a -1 (Tabela 1). O classificador k-means (Figura 9a) não demonstrou assertividade na diferenciação entre espécies invasoras (classe 2) e outras classes mapeadas. Pode ser observado que os pixels na cor marrom, os quais representam o solo exposto (classe 3), estão confusamente 
presentes nas áreas onde há presença quase que total de espécies invasoras e vegetação remanescente. Esta (na cor verde oliva), por sua vez, ocupou locais onde a presença majoritária era de espécies invasoras, conforme pode ser visualizado na área circundada com linha em cor vermelha no mosaico (Figura 9c). A identificação de áreas com espécies invasoras é incerta, pois contrasta com outras classes. A classificação NGRDI gerou valores de -1.00 a 1.00 , onde os valores negativos representam os locais onde há pouca ou quase nenhuma vegetação (solo exposto) e os valores próximos de 1 indicam maior presença de vegetação (maior área foliar) (Figura 9b). O índice NGRDI estima a presença de vegetação a partir da área foliar (ELAZAB et al., 2015), quanto maior forem os valores na escala positiva do índice NGRDI maior a presença de vegetação e de biomassa (ELAZAB et al., 2015; MOTOHKA et al., 2010; HUNT et al., 2005).

Tabela 1. Classe e respectivo valor de referência para o classificador k-means e o índice de vegetação NGRDI.

Tabela 1. Class and its reference value for the k-means classifier and the vegetation index NGRDI.

\begin{tabular}{lcc}
\hline Classe & $\begin{array}{c}\text { Classificador } \\
\text { (k-means) }\end{array}$ & $\begin{array}{c}\text { Índice Vegetação } \\
\text { (NGRDI) }\end{array}$ \\
\hline Vegetação Remanescente & 1 & 0,25 a 1 \\
Espécie Invasora & 2 & 0 a 0,25 \\
Solo Exposto & 3 & $-0,10$ a -1 \\
\hline
\end{tabular}

\subsection{Análise do modelo de dossel de espécies invasoras (CHIs)}

As alturas individuais das espécies invasoras estão ilustradas na Figura 10. Verifica-se na Figura 10a, que o ajuste do modelo para valores a partir de $49 \mathrm{~cm}$ mínimos e $110 \mathrm{~cm}$ máximos, acabam por remover muitas áreas em que há presença de espécies invasoras que foram confirmadas no trabalho de campo. Na Figura 10b, o modelo foi ajustado para valores mínimos de $20 \mathrm{~cm}$ e $140 \mathrm{~cm}$ para valores máximos, e finalmente obtiveram-se alturas que coincidem com a realidade, entretanto, fica claro que ainda assim houve uma diferença de altitude no modelo $\mathrm{CH}_{\mathrm{IS}}$. A altura das espécies invasoras variou de $20 \mathrm{~cm}$ a $140 \mathrm{~cm}$, e em algumas áreas houve valores inferiores ou superiores ao estimado em campo, indicando falhas ou erros que podem estar relacionados principalmente a menor sobreposição das imagens como também aos erros médios no eixo $\mathrm{Z}$ (altura).

Os pontos coletados em campo, com as medidas mensuradas manualmente, foram sobrepostos às áreas onde o modelo $\mathrm{CH}_{\mathrm{IS}}$ reconheceu como regiões com presença de espécies invasoras, compreendendo valores desde $20 \mathrm{~cm}$ até $140 \mathrm{~cm}$ (Figura 11). Nota-se que existe uma diferença (erro de estimativa) de até $60 \mathrm{~cm}$ em 29,7\% dos pontos. Em contrapartida, em outras regiões os valores são coincidentes com as medidas recolhidas em campo, principalmente na área amostral 1 (Figura 12a). Na área amostral 2 foi onde ocorreu maiores diferenças na altura do modelo (Figura 12b). a)

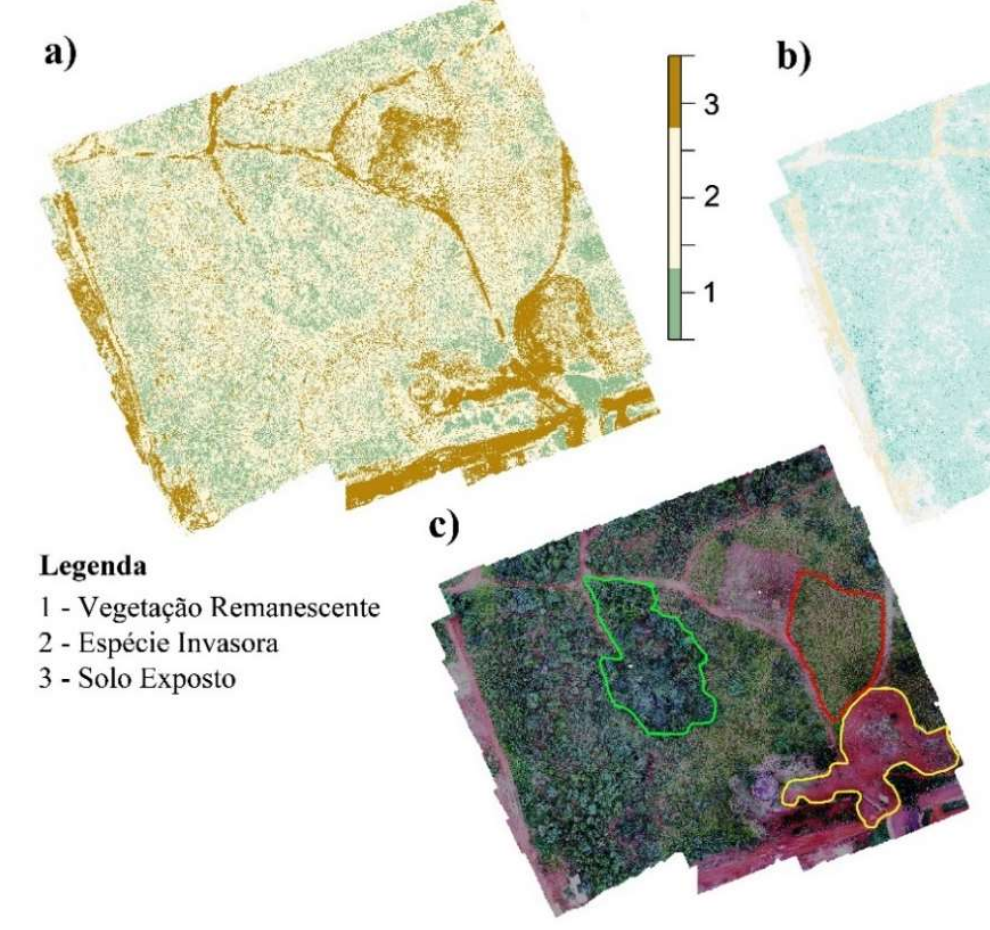

b)

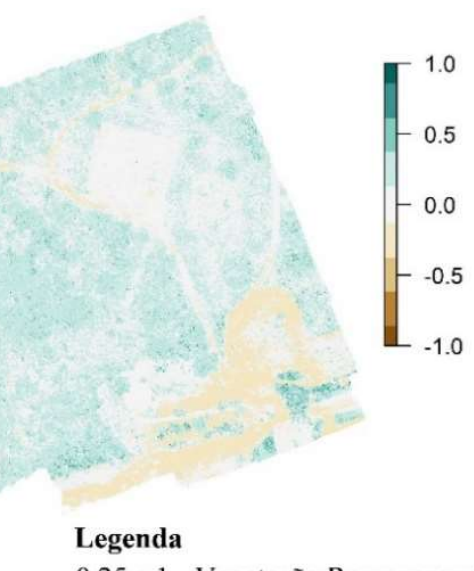

0.25 a 1 - Vegetação Remanescente 0 a 0.25 - Espécie Invasora

-0.10 a -1 - Solo Exposto

\section{Legenda

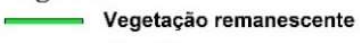 \\ Urochloa spp. \\ Solo exposto}

Figura 9. Classificação k-means com 3 diferentes classes (a); índice de vegetação NGRDI com escala de valores de -1 a 1 (b); mosaico para comparação (c).

Figure 9. k-means classification with 3 different classes (a); NGRDI vegetation index with a scale of values from -1 to 1 (b); mosaic for comparison (c).

\subsection{Avaliação da precisão do modelo $\mathrm{CH}_{\mathrm{IS}}$ em relação à classificação K-means e índice de vegetação NGRDI}

Os resultados do índice de vegetação NGRDI (Figura 13a) da área amostral 1 indicam que não está clara a distinção entre as classes de vegetação de porte arbóreo (vegetação remanescente) e vegetação gramínea (espécie invasora).
Existem alguns ruídos (pixels em cor azul) na classificação NGRDI, alguns locais representados por pixel azul $(0,5$ a 1,0$)$ relaciona-se à vegetação remanescente, porém em alguns locais onde há presença de pixels azuis, são locais onde predomina vegetação do porte gramíneo (Urochloa spp.), ou seja, locais onde houve oscilação na coloração da imagem classificada (ortomosaico), foi interpretado como sendo 
Pessi et al.

porte arbóreo, porém em campo constata-se que não há vegetação de grande porte, mas apenas espécies invasoras. $\mathrm{Na}$ Figura 13d estão circundados os locais onde há algumas espécies arbóreas misturadas com a Urochloa spp., os demais locais no índice de vegetação NGRDI que considerou como pixels na escala de 0,5 a 1 e não estão circundados no mosaico, considera-se como erro no logaritmo de classificação.
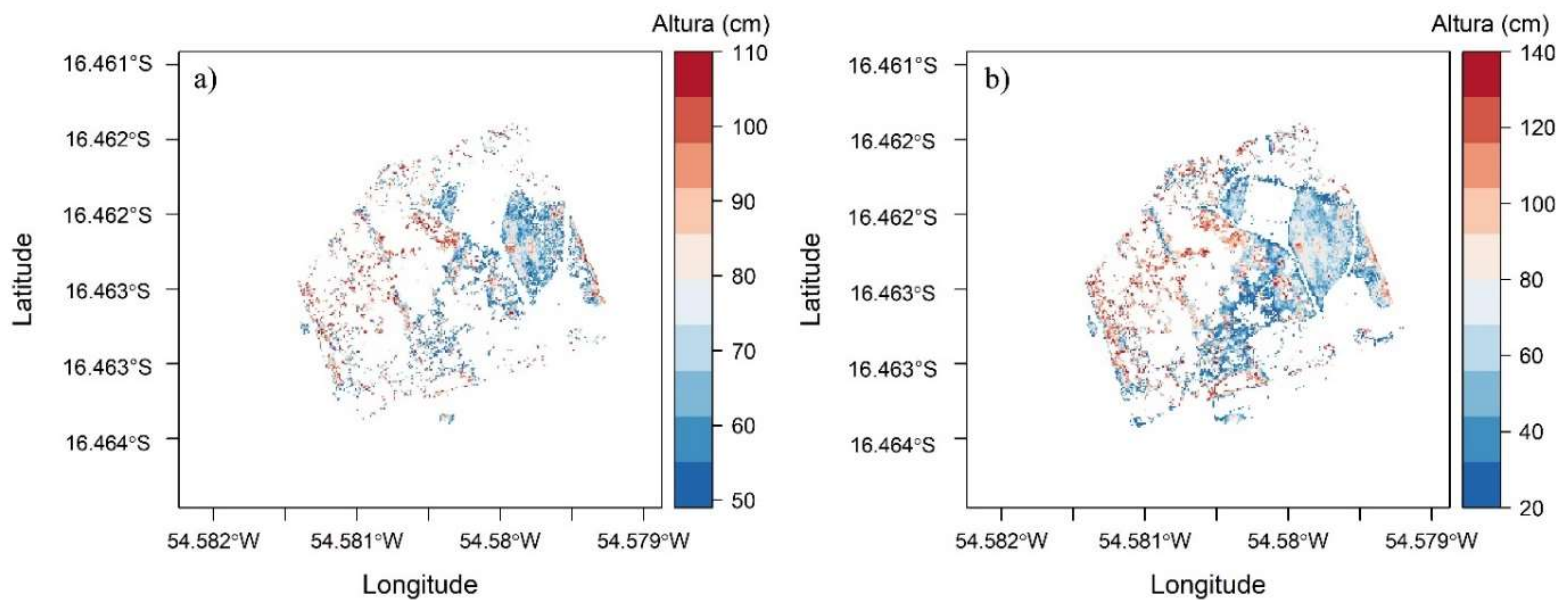

Figura 10. Modelo de altura de dossel de espécies invasoras $\left(\mathrm{CH}_{\mathrm{IS}}\right)$ com as alturas extraídas a partir do modelo $\mathrm{CHM}$. a) plantas que estiveram entre as medidas de $49 \mathrm{~cm}$ a $110 \mathrm{~cm}$; b) plantas com alturas de dossel a partir de $20 \mathrm{~cm}$ a $140 \mathrm{~cm}$.

Figure 10. Model of canopy height of invasive species $\left(\mathrm{CH}_{\mathrm{IS}}\right)$ with heights extracted from the $\mathrm{CHM}$ model. a) plants that were between the measures of $49 \mathrm{~cm}$ to $110 \mathrm{~cm}$; b) plants with canopy heights from $20 \mathrm{~cm}$ to $140 \mathrm{~cm}$.


Figura 11. $\mathrm{CH}_{\mathrm{IS}}$ com os pontos e medidas coletadas em campo para aferição do modelo.

Figure 11. $\mathrm{CH}_{\mathrm{IS}}$ with the points and measurements collected in the field to measure the model.

a)

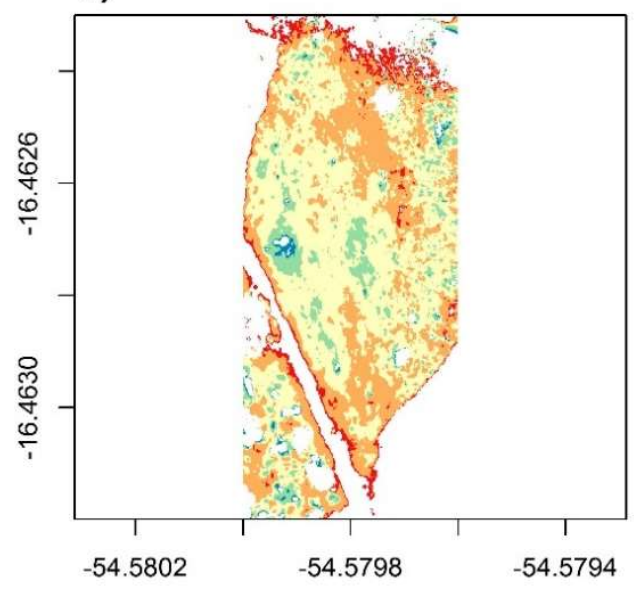

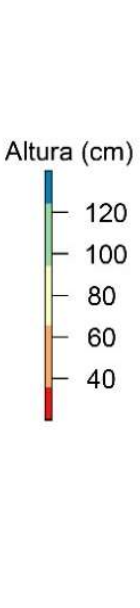

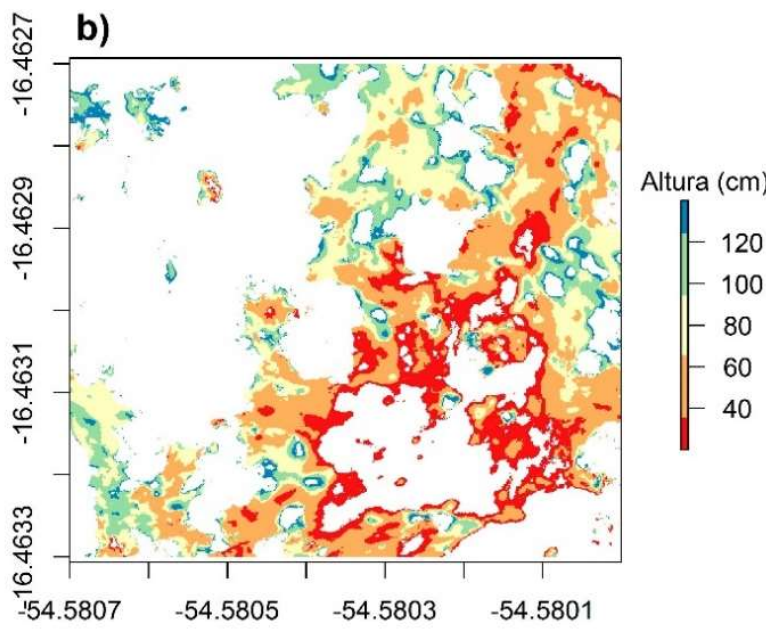

Figura 12. Em a) área amostral 1 e em b) área amostral 2, ambas usadas para verificar a precisão do modelo.

Figure 12 . In a) sample area 1 and in b) sample area 2 , both used to verify the accuracy of the model. 
O classificador k-means (Figura 13b) obteve resposta muito similar quanto a diferenciação dos extratos da vegetação quando comparado ao índice NGRDI, porém, assim como esse, ocorre grande quantidade de ruídos que dificultam a detecção de espécies invasoras em determinadas áreas de maneira precisa. As classes de solo exposto e espécies invasoras em algumas áreas se misturam, o que torna difícil saber se de fato naqueles locais há espécie invasora ou
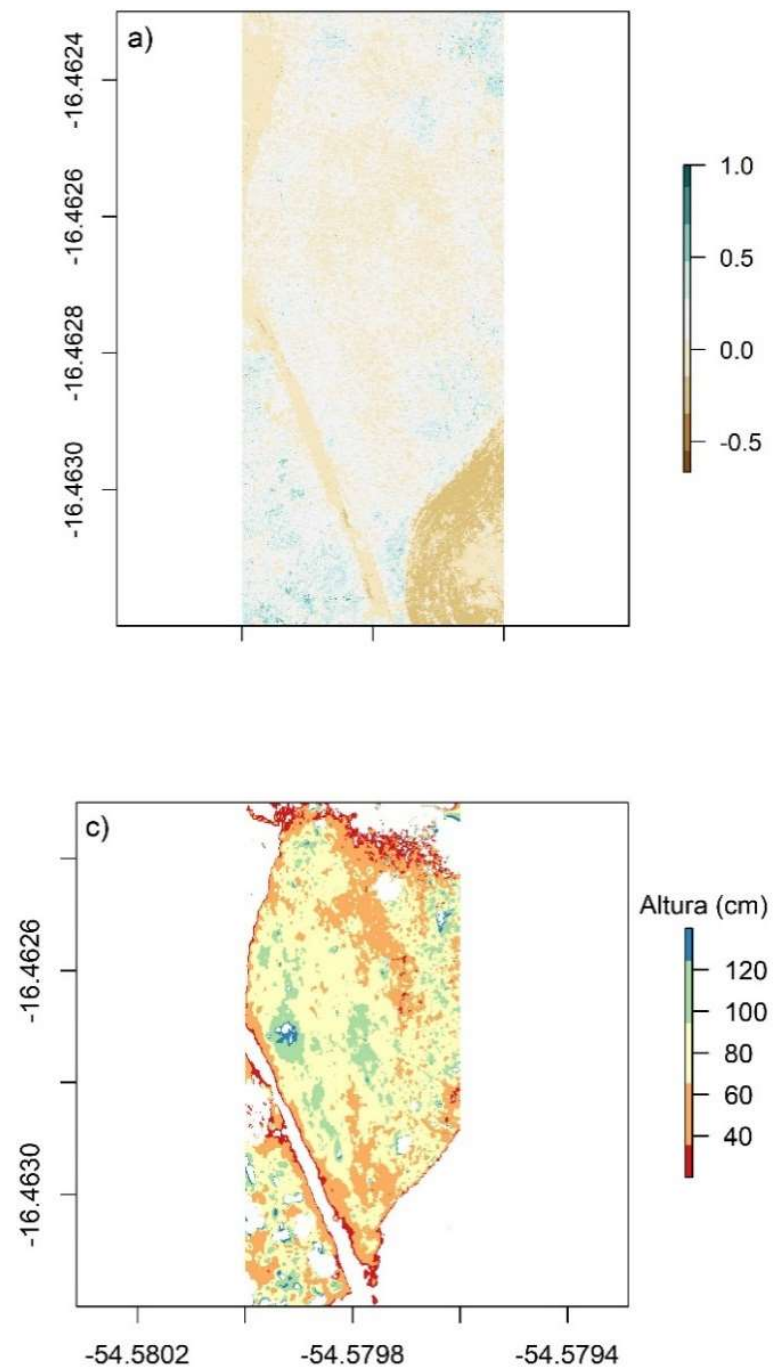

solo exposto (Figura 13b). Já o modelo $\mathrm{CH}_{\mathrm{IS}}$ (Figura 13c) permitiu a detecção de espécies invasoras mais precisamente, que foram classificadas a partir da altura do dossel (CHM), facilitando a diferenciação entre as três classes encontradas na área de estudo, que são a vegetação arbórea (vegetação remanescente), as espécies invasoras (Urochloa spp.), e o solo exposto.

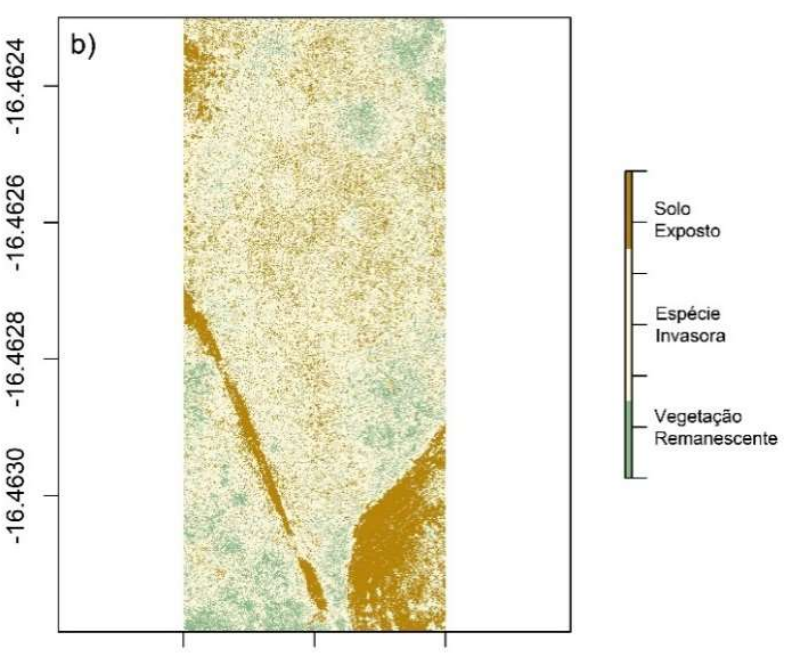

d)

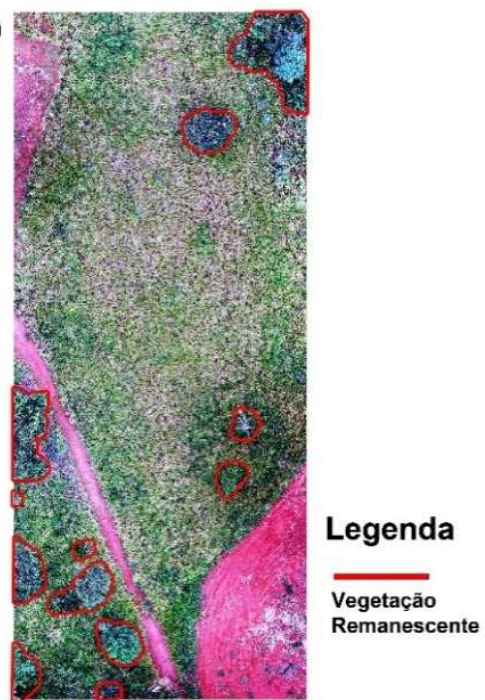

Figura 13. Amostra 1: análise comparativa entre índice de vegetação NGRDI (a), classificação k-means (b), e modelo CH $\mathrm{CH}_{\mathrm{IS}}$ Círculos em vermelho no mosaico (Figura d) representam fragmentos de vegetação remanescente.

Figure 13. Sample 1: comparative analysis between NGRDI vegetation index (a), k-means classification (b) and $\mathrm{CH}_{\mathrm{IS}}$ model. Circles in red in the mosaic (Figure d) represent fragments of remaining vegetation.

Os resultados da área amostral 2, mesmo que promissores, demonstram falhas de maneira similar no classificador k-means (Figura 14b) e no índice de vegetação NGRDI (Figura 14a) resultando no mesmo padrão de classificação que foi mencionado na área amostral 1, com pouca precisão quanto a diferenciação de vegetação remanescente de espécies invasoras. O modelo $\mathrm{CH}_{\mathrm{IS}}$ apresentou algumas falhas na identificação de espécies invasoras, principalmente na área demarcada com linha vermelha no mosaico (Figura 14d), local esse onde há alta presença de Urochloa spp. Em quase toda sua extensão, o modelo $\mathrm{CH}_{\mathrm{IS}}$ classificou relativamente bem partindo de uma análise visual, no entanto, na região inferior direita da área amostral 2, ocorreu ausência de informação, sendo que nesse quadrante (Figura 14c e 14d) existe a presença de espécies invasoras. Essa diferença no eixo Z (altura) está relacionada com os modelos MDS e MDT, pois existem erros na acurácia posicional, uma vez que não foi trabalhado com GPS geodésico (RTK), mas apenas com o GNSS do equipamento de voo (ARP).

No modelo $\mathrm{CH}_{\mathrm{IS}}$ para a área amostral 2, as informações que estão abaixo de $10 \mathrm{~cm}$ foram excluídas, tendo em vista 
que foi aplicado no modelo apenas valores a partir de $20 \mathrm{~cm}$ até $140 \mathrm{~cm}$. O que ocorreu neste caso foi que houve diferença quanto a elevação do terreno, erros no eixo $Z$ (elevação) entre $0,4 \mathrm{~m}$ a $1,2 \mathrm{~m}$ (Figura 15). Isto se deve principalmente pelo fato das informações de elevação (cota altimétrica) terem sido fornecidas apenas pelo GNSS da ARP, equipamento que não possui grande precisão quanto à elevação. Para tanto, seria necessário o uso de um RTK (Real Time Kinematic), que não foi utilizado para este trabalho. O erro médio para o eixo Z (altura) foi de $0,751895 \mathrm{~m}$, valor que corrobora com as diferenças encontradas no modelo $\mathrm{CH}_{\mathrm{IS}}$ comparado com os dados de campo. Os valores de erro do eixo X, Y e Z estão tabulados na Tabela 2.
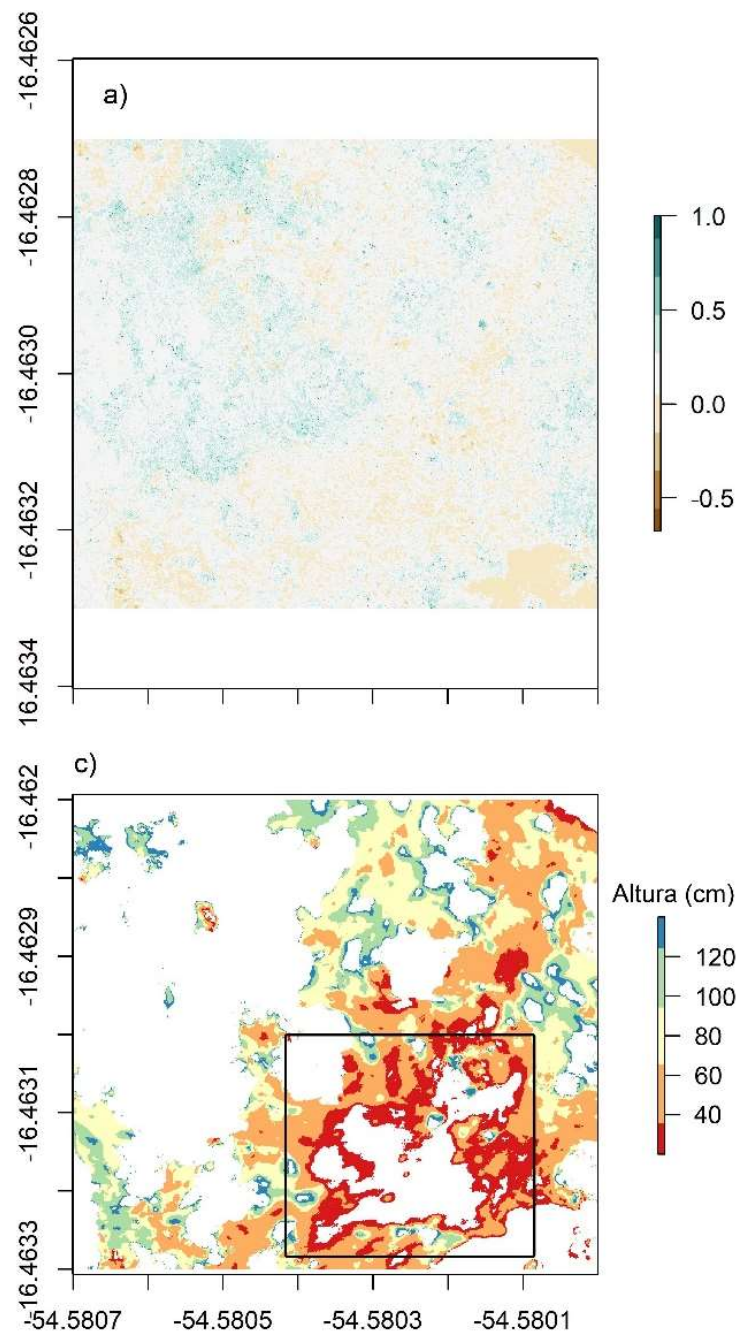

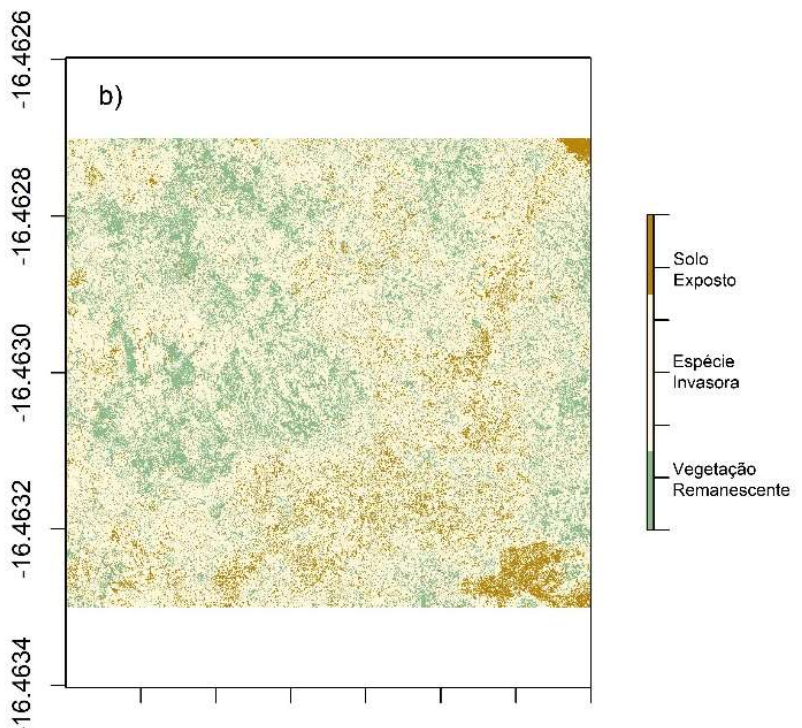

d)



Figura 14. Amostra 2: análise comparativa entre classificação k-means (a), índice de vegetação NGRDI (b), e modelo CHIs. Mosaico para observação na Figura 14d.

Figure 14. Figure 14. Sample 2: comparative analysis between k-means classification (a), vegetation index NGRDI (b), and model CHIs. Mosaic for observation in Figure 14d.

Tabela 2. Erro médio para os eixos X, Y e Z. X - Longitude, Y Latitude, Z - Altitude

Table 2. Average error for $\mathrm{X}, \mathrm{Y}$ and $\mathrm{Z}$ axes. $\mathrm{X}$ - Longitude, $\mathrm{Y}$ Latitude, Z - Altitude.

\begin{tabular}{ccccc}
\hline $\begin{array}{c}\text { Erro X } \\
(\mathrm{m})\end{array}$ & $\begin{array}{c}\text { Erro Y } \\
(\mathrm{m})\end{array}$ & $\begin{array}{c}\text { Erro Z } \\
(\mathrm{m})\end{array}$ & $\begin{array}{c}\text { Erro XY } \\
(\mathrm{m})\end{array}$ & $\begin{array}{c}\text { Erro Total } \\
(\mathrm{m})\end{array}$ \\
\hline 1,69044 & 0,799452 & 0,751895 & 1,86995 & 2,01546 \\
\hline
\end{tabular}

\subsection{Testes de acurácia, correlação e índice Kappa}

$\mathrm{O}$ modelo $\mathrm{CH}_{\mathrm{IS}}$ obteve alta precisão ao detectar a presença de espécies invasoras quando comparado com os demais modelos conforme pode ser visualizado na Tabela 3, os resultados de acurácia, Kappa e correlação de Pearson. A acurácia geral do modelo $\mathrm{CH}_{\mathrm{IS}}$ obteve valores de 0,973 na área amostral 1 e 0,9 na área amostral 2, e os valores de Kappa foram 0,88 (área amostral 1) e 0,751 (área amostral 2), o que indica que o modelo $\mathrm{CH}_{\mathrm{IS}}$ foi preciso e obteve resultados Kappa substanciais (VIEIRA; GARRETT, 2005; MCHUGH, 2012) principalmente na área amostral 1, onde visualmente o modelo permitiu discriminar as espécies invasoras em contraste com a vegetação remanescente. Já a área amostral 2, os valores foram menores por conta das áreas onde houve diferenças significativas na altura do modelo (Figura 14). A correlação de Pearson foi efetivamente alta (ASUERO et al., 2006) nas duas áreas amostrais do modelo $\mathrm{CH}_{\mathrm{IS}}$. Os menores valores de Kappa corresponderam ao modelo k-means $(-0,046$ e 0,123$)$ seguido 
do modelo NGRDI $(0,233$ e 0,127$)$. O maior número de erros (OER) foi observado na classificação k-means $(0,790$, área amostral 1). O fato de ocorrer um alto valor de erro nesse classificador se deve principalmente pela grande quantidade de ruídos (grande número de pixels espalhados) observados nesse modelo. O modelo NGRDI também apresentou um alto valor de erros na área amostral $2(0,482)$, devido à grande abrangência desse classificador na identificação da vegetação sem diferenciar as diferentes estruturas de dossel.

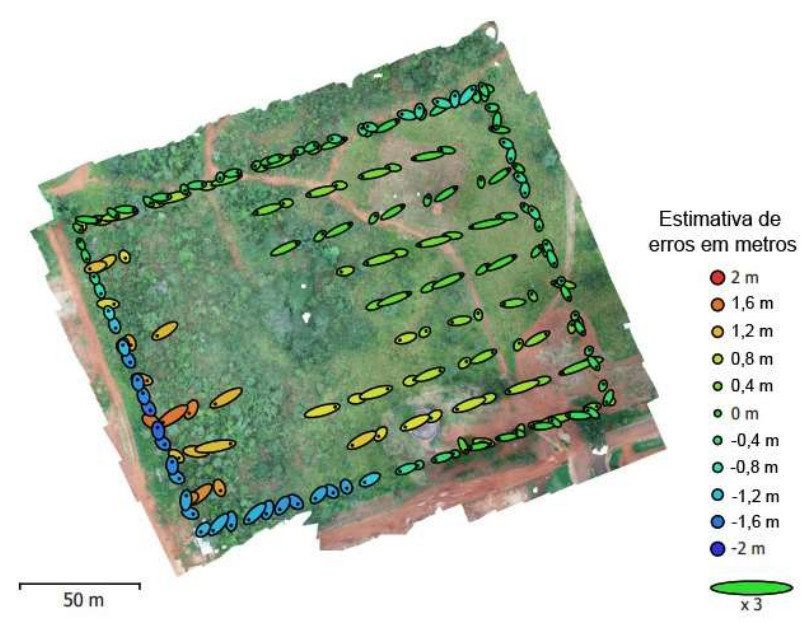

Figura 15. Localizações da câmera e estimativas de erros. O erro Z é representado pela cor da elipse. Os erros X, Y são representados pela forma de elipse. Os locais estimados da câmera são marcados com um ponto preto.

Figure 15. Camera locations and error estimates. The error $Z$ was represented by the color of the ellipse. The X, Y errors was represented by the ellipse form. Estimated camera locations are marked with a black dot.

Tabela 3. Valores de precisão obtidos para os modelos $\mathrm{CH}_{\mathrm{IS}}, \mathrm{K}$ means e NGRDI para cada uma das áreas amostrais do local de estudo. Coeficiente de correlação de Pearson (Spearman Correlation Coefficiente, SCC), Taxa de erro geral (Overall Error Rate, OER), especificidade (Specificity, SP), sensibilidade (Sensitivity, SN), acurácia geral (Overall Accuracy Rate, OAR).

Table 3. Precision values obtained for the $\mathrm{CH}_{\mathrm{IS}}$, $\mathrm{K}$-means and NGRDI models for each of the sample areas of the study site. Pearson correlation coefficient (Spearman Correlation Coefficiente, SCC), General Error Rate (OER), Specificity (SP), Sensitivity (SN), Overall Accuracy Rate (OAR).

\begin{tabular}{clcccccc}
\hline $\begin{array}{c}\text { Área } \\
\text { amostral }\end{array}$ & Modelo & SCC & OER & SP & SN & OAR & $\begin{array}{c}\text { Cohen's } \\
\text { Kappa }\end{array}$ \\
\hline \multirow{2}{*}{1} & CHIS & 0,888 & 0,026 & 0,895 & 0,986 & 0,973 & 0,888 \\
& K-means & $-0,128$ & 0,79 & 0,122 & 0,75 & 0,209 & $-0,046$ \\
& NGRDI & 0,235 & 0,204 & 0,316 & 0,898 & 0,795 & 0,233 \\
\hline \multirow{2}{*}{2} & CHIS & 0,776 & 0,1 & 0,871 & 1 & 0,9 & 0,751 \\
& K-means & 0,124 & 0,4 & 0,721 & 0,398 & 0,6 & 0,123 \\
& NGRDI & 0,156 & 0,482 & 0,775 & 0,378 & 0,518 & 0,127 \\
\hline
\end{tabular}

\section{DISCUSSÃO}

Nossos resultados ilustram que o sistema de sensores de ARP pode ser usado para coletar dados espaciais de áreas protegidas e ou campos de cultivo, que podem ser pósprocessados para derivar estimativas de altura do dossel capazes de diferenciar as estruturas da vegetação. As imagens da área amostral foram extraídas com base nos pontos de referência conhecidos do GNSS e as alturas das culturas foram então estimados pelo PhotoScan. O modelo proposto neste estudo $\left(\mathrm{CH}_{\mathrm{IS}}\right)$ mostrou ser mais eficiente para diferenciar a altura do dossel da vegetação quando comparado aos modelos de classificação por índice de vegetação NGRDI e classificador não superviosonado kmeans. O modelo $\mathrm{CH}_{\mathrm{IS}}$ mesmo apresentando limitações na estimativa da altura do dossel, por ter sido trabalhado apenas com o GNSS da ARP, demostrou bons resultados. Ocorre que o GNSS não possui alta precisão na estimativa dos eixos $\mathrm{x}, \mathrm{y}$ e $\mathrm{z}$, ocorrendo falhas ao ser aplicado o logaritmo do PhotoScan, como aconteceu nos resultados da Figura 6, onde há presença de valores negativos, apresentando oscilações na altura do terreno em locais onde não deveriam ter aquelas medidas apresentadas.

A abordagem para o mapeamento de espécies invasoras baseado na classificação não supervisionada k-means depende de observações in situ para validar se o classificador foi coerente com a realidade da área classificada. Neste sentido, este método demandaria mais tempo, recursos e maior imprecisão quando comparado ao método $\mathrm{CH}_{\mathrm{IS}}$, como pôde ser visualizado nos resultados da Tabela 3. Se observações in situ estiverem disponíveis para realizar a tarefa de rotulagem automática, o procedimento proposto poderia ser automatizado, fazendo com que o processo seja rápido. Os pontos de referência poderiam ser coletados com os Smart Applications em dispositivos móveis (BORDOGNA et al., 2016) ou fornecidos por voluntários como amostras de interpretação de imagens, como demonstrado em vários projetos colaborativos abertos em vários campos da ciência (FRANZONI; SAUERMANN, 2014). Um exemplo é o projeto Ground Truth 2.0, financiado pela Comissão Européia, que usa ciência cidadã para a gestão de terras e recursos naturais (GROUND TRUTH 2.0, 2017). Ainda assim, demandaria uma equipe maior no processo de coleta de dados e custos elevados nesse processo de classificação, ainda assim podendo ser menos preciso do que o modelo apresentado neste estudo.

Em relação ao desempenho do mapeamento de espécies invasoras a partir do índice NGRDI, a maior precisão de classificação foi de OAR 79\%, porém o Kappa foi baixo com 0,233 de acurácia. Isso demonstra que usar apenas a informação espectral da banda do visível (RGB), mesmo quando resumida em um índice de vegetação que use dessas bandas (NGRDI), não tem capacidade de diferenciar alvos de vegetação diferentes, minimizando assim a separabilidade entre classes de vegetação (espécies invasoras, vegetação remanescente), sendo que esse problema poderia ser minimizado com o uso de bandas multiespectrais, mas ainda assim não garantindo grande precisão na diferenciação. $\mathrm{O}$ índice de vegetação por diferença normalizada (NDVI) é amplamente utilizado na agricultura de precisão e na gestão ambiental (KYRATZIS et al., 2017; BAENA et al., 2017; YANG et al., 2017) por sua simplicidade (muitas vezes na ausência de uma avaliação quantitativa das alternativas), entretanto requer equipamento mais sofisticado (sensores multiespectrais) o que acarreta em elevar o custo do equipamento para realizar um trabalho como este. De fato, o NDVI é altamente sensível a mudanças abruptas do solo nu para a presença de culturas, quando há um forte contraste entre o solo e a planta em refletância do infravermelho próximo (NIR), como na fase pós-emergência (HUETE, 1988). Porém quando se trata de diferenciar os diferentes substratos da vegetação, o NDVI é deficiente. Em contraste, 
os índices que utilizam de bandas multiespectrais (índice de vegetação ajustado ao solo SAVI, NDVI e o índice de vegetação ajustado ao solo modificado MSAVI) forneceram resultados mais precisos do que o NGRDI quando usado para mapeamento direto de espécies invasoras (MATESE; DI GENNARO, 2018; WAN et al., 2018).

$\mathrm{O}$ baixo custo e a facilidade de criar visualizações $3 \mathrm{D}$ com imagens derivadas de drones (ARP) devem aumentar seu uso nos próximos anos. A possibilidade de uma rápida estimativa da altura do dossel da vegetação durante a recuperação de áreas protegidas com presença de espécies invasoras ou o crescimento da cultura agrícola fornecerá ao gestor ambiental e ao agricultor uma ferramenta para decidir prontamente sobre as estratégias de manejo do dossel e tomadas de decisão. Uma desvantagem do método $\mathrm{CH}_{\mathrm{IS}}$ para criar conjuntos de dados 3D com maior precisão é a necessidade do RTK, equipamento de difícil manuseio e de alto valor comercial, no entanto, informações coletadas a partir do GNSS do drone é uma opção viável, já que o algoritmo possui a capacidade em identificar a altura do dossel com precisão. Este estudo confirma a viabilidade de uma avaliação rápida preliminar da altura do dossel da vegetação obtida por imagens de alta resolução. Os métodos tradicionais de estimativa da altura do dossel usados pelos gestores ambientais e agricultores costumam consumir muito tempo e dinheiro. Por exemplo, a análise da madeira de poda e o comprimento e o peso da parte aérea são métodos de amostragem de solo que consomem tempo e são destrutivos. Esses métodos também exigem procedimentos difíceis em campo e em laboratório. Por outro lado, a avaliação da altura da copa usando imagens baseadas em RPA permite um método não destrutivo e espacial, benéfico para o meio ambiente.

\section{CONCLUSÕES}

Este estudo propõe um sistema para o mapeamento de espécies invasoras em áreas remanescentes e ou em recuperação via aeronaves remotamente pilotadas para projetar tratamentos de controle de espécies invasoras específicas do local a ser monitorado. Para os modelos convencionais (classificação não supervisionada e índices de vegetação) discriminarem as classes cultura, vegetação remanescente e espécies invasoras, torna-se difícil, pois essas têm propriedades espectrais muito semelhantes. O sistema proposto, $\mathrm{CH}_{\mathrm{IS}}$, faz uso de um algoritmo de segmentação e uma técnica de seleção de padrões baseados em altura da copa das plantas. Embora ambas (plantas remanescentes e espécies invasoras) apresentem propriedades espectrais muito semelhantes, o método é capaz de distingui-las com precisão usando apenas uma seleção de dados da altura da copa, características fáceis de calcular e pouquíssima intervenção do usuário na área. $\mathrm{O}$ uso de outras características de dados mostra grande melhoria quando comparado com a média comumente usada e desvio padrão na classificação de objetos. A representação gráfica dos resultados mostra que o uso da abordagem não é apenas promissor para a detecção de espécies invasoras emergentes em áreas em recuperação ambiental, mas também entre as linhas de cultura agrícola.

O método proposto abre um novo caminho para cenários ambientais e agronômicos e especialmente para a ciência de espécies invasoras. Como a intervenção do usuário é limitada, o principal gargalo deste sistema é evitado, tornando sua aplicação fácil na prática com o equipamento apropriado. Usando nossa abordagem, o especialista teria que intervir apenas no processo de aquisição de dados (por exemplo, voo com a ARP) e na rotulagem de dados (validando os resultados do algoritmo automático com dados de campo). O restante das etapas (mosaico de dados, segmentação de dados, cálculo de diferentes características e classificações) pode ser executado automaticamente sem a supervisão necessária. Nesse sentido, mapas de espécies invasoras podem ser facilmente produzidos a tempo para o tratamento pós-emergência precoce em áreas agrícolas como também em tempo de não prejudicar áreas em estado de recuperação.

\section{AGRADECIMENTOS}

O presente trabalho foi realizado com apoio da Coordenação de Aperfeiçoamento de Pessoal de Nível Superior - Brasil (CAPES) - Código de Financiamento 001 e do CNPq (Processo 441975/2018-6 e 315170/2018-2).

\section{REFERÊNCIAS}

ASUERO, A. G.; SAYAGO, A.; GONZÁLEZ, A. G. The Correlation Coefficient: An Overview. Critical Reviews in Analytical Chemistry, Boca Raton, v. 36, n. 1, p. 4159 , 2006. DOI: https://dx.doi.org/10.1080/10408340500526766

AGISOFT PHOTOSCAN PROFESSIONAL (Version 1.4.0) (Software). 2017. Available on: <http://www.agisoft.com/downloads/installer/>.

BAENA, S.; MOAT, J.; WHALEY, O.; BOYD, D. S. Identifying species from the air: UAVs and the very high resolution challenge for plant conservation. PLoS ONE, v. 12, p. e0188714, 2017. DOI: https://dx.doi.org/10.1371/journal.pone.0188714

BARRERO, O.; PERDOMO, S. A. RGB and multispectral UAV image fusion for Gramineae weed detection in rice fields. Precision Agriculture, Dordrecht, v. 19, p. 809822, 2018. DOI: https://dx.doi.org/10.1007/s11119017-9558-x

BIVAND, R.; TIM, K.; BARRY, R. Rgdal: Bindings for the 'Geospatial' Data Abstraction Library. R package version 1.3-4. 2018. Available on: https://CRAN.Rproject.org $/$ package $=$ rgdal

BORDOGNA, G.; KLIMENT, T.; FRIGERIO, L.; BRIVIO, P. A.; CREMA, A.; STROPPIANA, D.; BOSCHETTI, M.; STERLACCHINI, S. A Spatial Data Infrastructure Integrating Multisource Heterogeneous Geospatial Data and Time Series: A Study Case in Agriculture." ISPRS International Journal of GeoInformation, v. 5, n. 5, p. 1-27, 2016. DOI: https://dx.doi.org/10.3390/ijgi5050073

DAVID, L. C. G.; BALLADO, A. H. J. Vegetation Indices and Textures in Object-based Weed Detection from UAV imagery. In: IEEE International Conference on Control System, Computing and Engineering, 6., 2016, Penang. Annals... Penang, Malaysia, 2016. p. 25-27.

DVORÁK, P., MULLEROVÁ, J.; BARTALOS, T.; BRUNA, J. Unmanned Aerial Vehicles For Alien Plant Species Detection And Monitoring. In: The International Archieves Of The Photogrammetry. Remote Sensing and Spatial Information Sciences, 2015. Annals... v. XL1/W4, 2015. 
EVANS, L. J.; JONES, T. H.; PENG, K.; EVANS, M. N.; SAIMIN, S.; GOOSSENS, B. Use of drone technology as a tool for behavioral research: a case study of crocodilian nesting. Herpetol. Conserv. Biol., Herpetological Conservation and Biology, v. 10, n. 1, p. 90-98, 2015.

ELAZAB, A.; BORT, J.; ZHOU, B.; SERRET, M. D.; NIETO-TALADRIZ, M. T.; ARAUS, J. L. The combined use of vegetation indices and stable isotopes to predict durum wheat grain yield under contrasting water conditions. Agricultural Water Management, Amsterdam, v. 158, p. 196-208, 2015. DOI: https://dx.doi.org/10.1016/j.agwat.2015.05.003

FENG, Q.; LIU, J.; GONG, J. UAV remote sensing for urban vegetation mapping using random forest and texture analysis. Remote Sensing, v. 7, p. 1074-1094, 2015. DOI: https://dx.doi.org/10.3390/rs70101074

FRANZONI, C.; SAUERMANN, H. Crowd Science: The Organization of Scientific Research in Open Collaborative Projects. Research Policy, Amsterdam, v. 43, n. 1, p. 1-20, 2014. DOI: https://dx.doi.org/10.1016/j.respol.2013.07.005

GINI, R.; SONA, G.; RONCHETTI, G.; PASSONI, D.; PINTO, L. Improving Tree Species Classification Using UAS Multispectral Images and Texture Measures. ISPRS International Journal of Geo-Information, v. 7, n. 8, p. 315, 2018.2 DOI: https://dx.doi.org/10.3390/iigi7080315

GITELSON, A. A.; KAUFMAN, Y. J.; STARK, R. et al. Novel algorithms for remote estimation of vegetation fraction. Remote Sensing of Environment, v.80, p.76$87,2002$.

GROUND TRUTH 2.0. 2017. Land Use Mapper. Disponível em: <http://gt20.eu/land-usemapper/>. Último acesso em: 30 nov. de 2018.

GOKTOGAN, A. H.; SUKKARIEH, S.; BRYSON, M.; RANDLE, J.; LUPTON, T.; HUNG, C. A rotary-wing unmanned air vehicle for aquatic weed surveillance and management. Journal of Intelligent and Robotic Systems volume, v. 57, p. 467-484, 2010. DOI: https://dx.doi.org/10.1007/s10846-009-9371-5

HARDIN, P. J.; JACKSON, M. W.; ANDERSON, V. J.; JOHNSON, R. Detecting Squarrose Knapweed (Centaurea virgata Lam. Ssp. squarrosa Gugl.) using a Remotely Piloted Vehicle: A Utah case study. GIsci. Remote Sensing, v. 44, n. 3, p. 203-219, 2007. DOI: https://dx.doi.org/10.2747/1548-1603.44.3.203

HIJMANS, R. J. Raster: Geographic Data Analysis and Modeling. R package version 2.8-19 2018. Available on: https://CRAN.R-project.org/package $=$ raster.

HYNDMAN, R.; ATHANASOPOULOS, G.; BERGMEIR, C.; CACERES, G.; CHHAY, L.; O'HARA-WILD, M.; PETROPOULOS, F.; RAZBASH, S.; WANG, E.; YASMEEN, F._forecast: Forecasting functions for time series and linear models_. R package version 8.5. 2019. Available

on: <URL:http://pkg.robjhyndman.com/forecast>.

HUETE, A. R. A Soil-Adjusted Vegetation Index (SAVI). Remote Sensing of Environment, Amsterdam, v. 25, n .3, p. 295-309, 1988 . DOI: https://dx.doi.org/10.1016/0034-4257(88)90106-X

HUNG, C.; XU, Z.; SUKKARIEH, S. Feature Learning Based Approach for Weed Classification Using High-
Resolution Aerial Images from a Digital Cameral Mounted on a UAV. Remote Sensing, v. 6, n. 12, p. 12037-12054, 2014.2 DOI: https://dx.doi.org/10.3390/rs61212037

HUNT, E. R. JR.; CAVIGELLI, M.; DOUGHTRY, C. S. T.; MCMURTREY III, J.; WALTHALL, C. L. Evaluation of Digital Photography from Model Aircraft for Remote Sensing of Crop Biomass and Nitrogen Status. Precision Agriculture, Dordrecht, v. 6, p. 359-378, 2005. DOI: https://dx.doi.org/10.1007/s11119-005-2324-5

IVOSEVIC, B.; HAN, Y.; CHO, Y.; KWON, O. The use of conservation drones in ecology and wildlife research. Journal of Ecology and Environment, v. 38, n. 1, p. 113-118, 2015.2 DOI: https://dx.doi.org/10.5141/ecoenv.2015.012

KANEKO, K.; NOHARA, S. Review of Effective Vegetation Mapping Using the UAV (Unmanned Aerial Vehicle) Method. Journal of Geographic Information System, v. 6, p. 733-742, 2014. DOI: https://dx.doi.org/10.4236/jgis.2014.66060

KYRATZIS, A. C.; SKARLATOS, D. P.; MENEXES, G. C.; VAMVAKOUSIS, V. F.; KATSIOTIS, A. Assessment of Vegetation Indices Derived by UAV Imagery for Durum Wheat Phenotyping under a Water Limited and Heat Stressed Mediterranean Environment. Frontiers in Plant Science, v. 8, n. 1114, p. 1-14, 2017. DOI: https://dx.doi.org/10.3389/fpls.2017.01114

$\mathrm{KOH}, \mathrm{L}$. P.; WICH, S. A. Dawn of drone ecology: low-cost autonomous aerial vehicles for conservation. Tropical Conservation Science, v. 5, n. 2, p. 121-132, 2012. DOI: https://dx.doi.org/10.1177\%2F194008291200500202

KNOTH, C.; KLEIN, B.; PRINZA, T.; KLEINBECKER, T. Unmanned Aerial Vehicles as Innovative Remote Sensing Platforms for High-Resolution Infrared Imagery to Support Restoration Monitoring in Cut-Over Bogs. Applied Vegetation Science, v. 16, n. 3, p. 509-517, 2013. DOI: https://dx.doi.org/10.1111/avsc.12024

KUHN, M. WING, J.; WESTON, S.; WILLIAMS, S.; KEEFER, S.; ENGELHARDT, A.; COOPER, T.; MAYER, Z.; KENKEL, B.; BENESTY, M.; LESCARBEAU, R.; ZIEM, A.; SCRUCCA, L.; TANG, Y.; CANDAN, C.; HUNT, T. Caret: Classification and Regression Training. R package version 6.0-81. 2018. Available on: https://CRAN.Rproject.org $/$ package $=$ caret

LALIBERTE, A. S.; GOFORTH, M. A.; STEELE, C. M.; RANGO, A. Multispectral Remote Sensing from Unmanned Aircraft: Image processing workflows and applications for rangeland environment. Remote Sensing, v. 3, n. 11, p. 2529-2551, 2011. DOI: https://dx.doi.org/10.3390/rs3112529

LEHMANN, J. R. K.; PRINZ, T.; ZILLER, S. R.; THIELE, J.; HERINGER, G.; MEIRA-NETO, J. A. A.; BUTTSCHARDT, T. K. Open-source processing and analysis of aerial imagery acquired with a low-cost unmanned aerial system to support invasive plant management. Frontiers in Environmental Science, v. 5, n. 44, p. 1-16, 2017. DOI: https://dx.doi.org/10.3389/fenvs.2017.00044

LI, W.; NIU, Z.; CHEN, H.; LI, D.; WU, M.; ZHAO, W.; Remote estimation of canopy height and aboveground biomass of maize using high-resolution stereo images from a low-cost unmanned aerial vehicle system. 
Ecological Indicators, v. 67, p. 637-648, 2016. DOI: https://dx.doi.org/10.1016/j.ecolind.2016.03.036

LUCIEER, A.; ROBINSON, S.; TURNER, D.; HARWIN, S.; KELCEY, J. Using a micro-UAV for ultra-high resolution multi-sensor observations of Antarctic moss beds. In: Proceedings of the ISPRS Congress, 22., 2016, Melbourne. Annals... Melbourne, Australia, 2012. p. 429-433,

MARTIN, F.; MULLERONÁ, J.; BORGNIET, L.; DOMMANGET, F.; BRETON, V.; EVETTE, A. Using Single- and Multi-Date UAV and Satellite Imagery to Accurately Monitor Invasive Knotweed Species. Remote Sensing, v. 10, n. 10 , p. 1662, 2018. DOI: https://dx.doi.org/10.3390/rs10101662

MATESE, A.; GENNARO, S. F. D.; BERTON, A. Assessment of a canopy height model (CHM) in a vineyard using UAV-based multispectral imaging. International Journal of Remote Sensing, Basingstoke, v. 38, n. 8, p. 2150-2160, 2017. DOI: https://dx.doi.org/10.1080/01431161.2016.1226002

MAECHLER, M.; ROUSSEEUW, P.; STRUYF, A.; HUBERT, M.; HORNIK, K. cluster: Cluster Analysis Basics and Extensions. R package version 2.0.7-1. 2018.

MATESE, A.; GENNARO, S. F. D. Practical Applications of a Multisensor UAV Platform Based on Multispectral, Thermal and RGB High Resolution Images in Precision Viticulture. Agriculture, v. 8, n. 7, p.1-13, 2018. DOI: https://dx.doi.org/10.3390/agriculture8070116

MANGIAFICO, S. rcompanion: Functions to Support Extension Education Program Evaluation. R package version 2.1.1. 2019. Available on: <https://CRAN.Rproject.org $/$ package $=$ rcompanion $>$.

MCHUGH, M. L. Interrater reliability: the kappa statistic. Biochemia Medica, v. 22, n. 3, p. 276-282, 2012. DOI: https://dx.doi.org/10.11613/BM.2012.031

MICHEZ, A.; PIÉGAY, H.; JONATHAN, L.; CLAESSENS, H.; LEJEUNE, P. Mapping of Riparian Invasive Species with Supervised Classification of Unmanned Aerial System (UAS) Imagery. International Journal of Applied Earth Observation and Geoinformation, Enschede, v. 44, n. 1, p. 88-94, 2016. DOI: https://dx.doi.org/10.1016/j.jag.2015.06.014

MOTOHKA, T.; NASAHARA, K. N.; OGUMA, H.; TSUCHIDA, S. Applicability of Green-Red Vegetation Index for Remote Sensing of Vegetation Phenology. Remote Sensing, v. 2, p. 2369-2387, 2010. DOI: https://dx.doi.org/10.3390/rs2102369

MÜlLEROVÁ, J.; BRUNA, J.; BARTALOS, T.; DVORÁK, P.; VÍTKOVÁ, M.; PYSÉK, P. Timing Is Important: unmanned aircraft vs. Satellite Imagery in Plant Invasion Monitoring. Frontiers in Plant Science, v. $8, \quad$ n. 887 , p. 1-13, 2017. DOI: https://dx.doi.org/10.3389/fpls.2017.00887

NEUWIRTH, E. RColorBrewer: ColorBrewer Palettes. R package version 1.1-2. 2014. Available on: <https:/ CRAN.Rproject.org $/$ package $=$ RColorBrewer $>$.

PEEL, M. C.; FINLAYSON, B. L.; MCMAHON, T. A. Updated world map of the K"oppen-Geiger climate classification. Hydrology and Earth System Sciences, v. 11, n. 5, p. 1633-1644, 2007. DOI: https://dx.doi.org/10.5194/hess-11-1633-2007
PEÑA, J. M.; TORRES-SÁNCHEZ, J.; DE CASTRO, A. I.; KELLY, M.; LÓPEZ-GRANADOS, F. Weed Mapping in Early-Season Maize Fields Using Object-Based Analysis of Unmanned Aerial Vehicle (UAV) Images. PLoS ONE, v. 8, n. 10, p. e77151, 2013. DOI: htps://dx.doi.org/10.1371/journal.pone.0077151

PENG, Z. R.; WANG, D.; WANG, Z.; GAO, Y.; LU, S. A study of vertical distribution patterns of PM2.5 concentrations based on ambient monitoring with Unmanned Aerial Vehicles: A case in Hangzhou, China. Atmospheric Environment, v. 123, p. 357-369, 2015. DOI:

https://dx.doi.org/10.1016/j.atmosenv.2015.10.074

R CORE TEAM. R: A language and environment for statistical computing. $\mathrm{R}$ Foundation for Statistical Computing, Vienna, Austria, 2018. Available on: $<$ https://www.R-project.org/>.

RANGO, A.; LALIBERTE, A. S.; HERRICK, J. E.; WINTERS, C.; HAVSTAD, K.; STEELE, C.; BROWNING, D. Unmanned aerial vehicle-based Remote Sensing for rangeland assessment, monitoring, and management. Journal of Applied Remote Sensing, v. 3 , n. 1 , p. 1-15, 2009. DOI: https://dx.doi.org/10.1117/1.3216822

SÁ, N. C. de; CASTRO, P.; CARVALHO, S.; MARCHANTE, E.; LÓPEZ-NÚNEZ, F. A.; MARCHANTE, H. Mapping the Flowering of an Invasive Plant Using Unmanned Aerial Vehicles: Is There Potential for Biocontrol Monitoring?. Frontiers in Plant Science, v. 9, p. 283, 2018. DOI: https://dx.doi.org/10.3389/fpls.2018.00293

STARK, D.; VAUGHAN, I. P.; EVANS, L. J.; KLER, H.; GOOSSENS, B. Combining drones and satellite tracking as an effective tool for informing policy change in riparian habitats: a proboscis monkey case study. Remote Sensing in Ecology and Conservation, v. 4, n. 1, p. 4452, 2018. DOI: https://dx.doi.org/10.1002/rse2.51

SOUZA, A. P.; MOTA, L. L.; ZAMADEI, T.; MARTIM, C. C.; ALMEIDA, F. T., PAULINO, J. Classificação climática e balanço hídrico climatológico no Estado de Mato Grosso. Nativa, Sinop, v. 1, n. 1, p. 34-43, 2013.

STROPPIANA, D.; VILLA, P.; SONA, G.; RONCHETTI, G.; CANDIANI, G.; PEPE, M.; BUSETTO, L.; MIGLIAZZI, M.; BOSCHETTI, M. Early season weed mapping in rice crops using multi-spectral UAV data. International Journal of Remote Sensing, Basingstoke, v. 39, n. 15-16, p. 5432-5452, 2018. DOI: https://dx.doi.org/10.1080/01431161.2018.1441569

STABLER, B. shapefiles: Read and Write ESRI Shapefiles. $\mathrm{R}$ package version 0.7. 2013. Available on: $<$ https://CRAN.R-project.org/package=shapefiles $>$.

TURNER, D.; LUCIEER, A.; MALENOVSKY, Z.; KING, D. H.; ROBINSON, S. A. Spatial co-registration of ultrahigh resolution visible, multispectral and thermal images acquired with a micro-UAV over Antarctic moss beds. Remote Sensing, v. 6, n. 5, p. 4003-4024, 2014. DOI: https://dx.doi.org/10.3390/rs6054003

VIEIRA, A. J.; GARRET, J. M. Understanding Interobserver Agreement: The Kappa Statistic. Family Medicine, Northfield, v. 37, n. 5, p. 360-363, 2005.

VISHWANATH, N.; RAMESH, B.; SREENIVASA, R. P. Unsupervised classification of remote sensing images using $\mathrm{K}$-means algorithm. International Journal of 
Latest Trends in Engineering and Technology (IJLTET), v. 7, n. 2, p.548-552, 2016.

VILJANEN, N.; HONKAVAARA, E.; NASI, R.; HAKALA, T.; NIEMELAINEN, O.; KAIVOSOJA, J. A Novel Machine Learning Method for Estimating Biomass of Grass Swards Using a Photogrammetric Canopy Height Model, Images and Vegetation Indices Captured by a Drone. Agriculture, v. 8, n. 5, p. 70, 2018. DOI: https://dx.doi.org/10.3390/agriculture8050070

WAN, H.; WANG, Q.; JIANG, D.; FU, J.; YANG, Y.; LIU, X. 2014. Monitoring the Invasion of Spartina Alterniflora Using Very High Resolution Unmanned Aerial Vehicle Imagery in Beihai, Guangxi (China). The Scientific World Journal, v. 2014, p. 1-7, 2014. DOI: https://dx.doi.org/10.1155/2014/638296

WAN, L.; LI, Y.; CEN, H.; ZHU, J.; YIN, W.; WU, W.; ZHU, H.; SUN, D.; ZHOU, W.; HE, Y. Combining UAV-Based Vegetation Indices and Image Classification to Estimate Flower Number in Oilseed Rape. Remote Sensing, v. 10, n. 9, p. 1-18, 2018. DOI: https://dx.doi.org/10.3390/rs10091484

YANG, G.; LIU, J.; ZHAO, C.; LI, Z.; HUANG, Y.; YU, H.; XU, B.; YANG, X.; ZHU, D.; ZHANG, X.; ZHANG, R.; FENG, H.; ZHAO, X.; LI, Z.; LI, H.; YANG, H. Unmanned Aerial Vehicle Remote Sensing for FieldBased Crop Phenotyping: Current Status and Perspectives. Frontiers in Plant Science, v. 8, n. 1111, p. $1-26,2017.2$ DOI: https://dx.doi.org/10.3389/fpls.2017.01111

ZAMAN, B., A. M. JENSEN, MCKEE, M. Use of HighResolution Multi-Spectral Imagery Acquired with an Autonomous Unmanned Aerial Vehicle to Quantify the Spread of an Invasive Wetlands Species. In: Proc. IEEE Int. Geoscience and Remote Sensing Symp. (IGARSS), 2011. Annals... 2011. p. 803-806. DOI: https://dx.doi.org/10.1109/igarss.2013.6723680

ZILIANI, M. G.; PARKES, S. D.; HOTEIT, I.; MCCABE, M. F. Intra-Season Crop Height Variability at Commercial Farm Scales Using a Fixed-Wing UAV. Remote Sensing, v. 10, n. 12, p.2007, 2018. DOI: https://dx.doi.org/10.3390/rs10122007

ZHANG, Q.; QIN, R.; HUANG, X.; FANG, Y.; LIU, L. Classification of ultra-high resolution orthophotos combined with DSM using a dual morphological top hat profile. Remote Sensing, v. 7, n. 12, p. 16422-6440, 2015. DOI: https://dx.doi.org/10.3390/rs71215840 